\title{
From linear to branching-time temporal logics: transfer of semantics and definability
}

\author{
Valentin Goranko* and Alberto Zanardo ${ }^{\dagger}$
}

October 24, 2006

\begin{abstract}
This paper investigates logical aspects of combining linear orders as semantics for modal and temporal logics, with modalities for possible paths, resulting in a variety of branching time logics over classes of trees. Here we adopt a unified approach to the Priorean, Peircean and Ockhamist semantics for branching time logics, by considering them all as fragments of the latter, obtained as combinations, in various degrees, of languages and semantics for linear time with a modality for possible paths. We then consider a hierarchy of natural classes of trees and bundled trees arising from a given class of linear orders and show that in general they provide different semantics. We also discuss transfer of definability from linear orders to trees and introduce a uniform translation from Priorean to Peircean formulae which transfers definability of properties of linear orders to definability of properties of all paths in trees.
\end{abstract}

\section{Introduction}

Linear time vs branching time is a major issue in temporal logic. In the former, time is viewed as a linearly ordered set of moments (time instants)

\footnotetext{
* School of Mathematics, University of Witwatersrand, Johannesburg. E-mail: goranko@maths.wits.ac.za.

$\dagger$ Department of Pure and Applied Mathematics, University of Padova. E-mail azanardo@math. unipd.it.
} 
(see e.g. $[1,9]$ ), while in the latter, time is pictured in a tree-like fashion: the past of any moment is linearly ordered, but there might be incomparable moments in its future (see e.g. $[3,5]$ ). In this view, every actual or conceivable course of the future is modelled as a linear time flow, and therefore linear orders play a crucial role in logics for branching time, too. Prior's Ockhamist and Peircean semantics for branching time [11], in fact, involve quantification over paths (or histories) in tree-like structures, where paths are maximal linearly ordered sets of moments. Moreover, this quantification can be viewed as (and in the Ockhamist logic it is) the result of the application of a modal operator (see e.g. [16]). This means that the languages and semantics for branching time can be obtained as combinations, in various degrees, of languages and semantics for linear time with a modality for possible paths.

In the paper we present this approach to logics for branching time, and study its aspects related to semantics and transfer of definability from linear orders to trees. The paper is structured as follows. In Section 2 we identify some important classes of linear orders which will be used in the paper. In Section 3 we study the topological closure of a class of linear orders obtained by adding emerging orders in bundles over that class, and introduce several natural classes of trees and bundled trees associated with a given class of linear orders. In Section 4 we present the Priorean and Peircean modal and temporal logics as fragments of the Ockhamist logic obtained as combinations of the linear time operators with the modal operators for possible paths, thus adopting a unified approach to Priorean, Peircean, and Ockhamist semantics of branching time logics. We discuss the expressiveness of these fragments with respect to the classes of trees and bundled trees introduced in the previous section and, in particular, show that the Peircean fragment suffices to distinguish them all. In Section 5 we discuss transfer of definability from linear orders to trees and introduce a uniform translation from Priorean to Peircean formulae which transfers definability of properties of linear orders to definability of properties of all paths in trees. In the last Section 7 we state and discuss a number of open problems and directions for further research. 


\section{Preliminaries on linear orders}

A strict linear order is a pair $(X,<)$ in which $<$ is a transitive and irreflexive relation on the non-empty set $X$, such that either $x<y$ or $y<x$ for all $x \neq y$ in $X$. Here we list some important classes of linear orders, used further in the paper:

$\mathcal{C}_{\text {lin }}$ : the class of all linear orders;

$\mathcal{C}_{\text {fin }}$ : the class of all finite linear orders;

$\mathcal{C}_{\text {first }}$ : the class of all linear orders with first element;

$\mathcal{C}_{\text {last }}$ : the class of all linear orders with last element;

$\mathcal{C}_{\text {udiscr }}$ : the class of all upwards discrete linear orders, i.e., those in which every non-last point has an immediate successor;

$\mathcal{C}_{\text {ddiscr }}$ : the class of all downwards discrete linear orders, i.e., those in which every non-first point has an immediate predecessor;

$\mathcal{C}_{\text {discr }}=\mathcal{C}_{\text {udiscr }} \cap \mathcal{C}_{\text {ddiscr }}$ : the class of all discrete linear orders;

$\mathcal{C}_{\text {dense }}$ : the class of all dense linear orders;

$\mathcal{C}_{\text {Ded }}$ : the class of all Dedekind complete linear orders;

$\mathcal{C}_{\text {cont }}$ : the class of all continuous (i.e., dense and Dedekind complete) linear orders;

$\mathcal{C}_{\mathrm{wf}}$ : the class of all well-founded linear orders;

$\mathcal{C}_{\text {iwf }}$ : the class of all inversely well-founded linear orders;

Note that $\mathcal{C}_{\text {fin }}=\mathcal{C}_{\text {wf }} \cap \mathcal{C}_{\text {iwf }}$.

The class of all linear orders isomorphic to a given linear order will be called an isomorphism type of linear orders, or simply a linear order type.

The following isomorphism types are of particular importance: $\omega$, of the natural numbers; $\omega^{*}$, of the negative integers; $\zeta$, of all integers; $\eta$, of the rationals, $\lambda$, of the reals.

The sum of two linear order types $l_{1}$ and $l_{2}$ is the linear order type $l_{1}+l_{2}$ with a domain consisting of a disjoint union of linear orders $\mathrm{L}_{1}=\left\langle X_{1},<_{1}\right\rangle$ and $\mathrm{L}_{2}=\left\langle X_{2},<_{2}\right\rangle$ of order types $\mathrm{l}_{1}$ and $\mathrm{l}_{2}$ respectively, and order relation $<$ which agrees with $<_{1}$ and $<_{2}$ on $\mathrm{L}_{1}$ and $\mathrm{L}_{2}$ respectively, and such that $x_{1}<x_{2}$ whenever $x_{1} \in \mathrm{L}_{1}$ and $x_{2} \in \mathrm{L}_{2}$. The sum $\mathcal{C}_{1}+\mathcal{C}_{2}$ is the class of all sums of an element of $\mathcal{C}_{1}$ with an element of $\mathcal{C}_{2}$.

An order embedding $f$ of a linear order $\mathrm{L}_{1}$ into the linear order $\mathrm{L}_{2}$ is 
cofinal whenever, for every $x_{2}$ in $\mathrm{L}_{2}$, there exists an $x_{1}$ in $\mathrm{L}_{1}$ such that $x_{2} \leq_{2} f\left(x_{1}\right)$. The cofinality type of a linear order $\mathrm{L}$ is the smallest ordinal which can be cofinally embedded in L. Every cofinality type is a cardinal and, by the Axiom of Choice, the cofinality type of $\omega_{\alpha}$ is $\omega_{\alpha}$ itself whenever $\alpha$ is a successor ordinal. Each of $\mathbb{N}, \mathbb{Z}, \mathbb{Q}$, and $\mathbb{R}$ has a cofinality type $\omega$.

\section{From linear orders to trees}

In the context of this paper, a tree is a strict order $T=\langle T, \prec\rangle$ with the left-linearity property: if $x \prec y$ and $z \prec y$, then either $x \prec z$, or $z \prec x$, or $x=z$. Moreover, we assume also that trees are connected, that is, for any $x, y$ in $\mathrm{T}$, there is a $z$ such that $z \preceq x$ and $z \preceq y$. A path in the tree $\mathrm{T}$ is a subset of $T$ linearly ordered by $\prec$, which is maximal for inclusion. The set of all paths in the tree $\mathrm{T}$ will be written as $\mathrm{H}(\mathrm{T})$ and the set of paths passing through $t$ will be written as $\mathrm{H}_{t}(\mathrm{~T})$. The elements of $\mathrm{H}_{t}(\mathrm{~T})$ will be sometimes called $t$-paths.

A more general structure for branching time is based on the notion of a bundle of admissible paths in a tree $\mathrm{T}$, where not necessarily all paths from $\mathrm{H}(\mathrm{T})$ are considered possible or acceptable, but only those in the bundle. For instance, in a computation tree (see e.g. $[7,15]$ ) one may wish to ban all computation runs which do not satisfy certain fairness conditions, and thus only consider the bundle of 'fair computations'.

Formally, we consider trees together with bundles on them, that is, sets $\mathrm{B} \subseteq \mathrm{H}(\mathrm{T})$ such that every point $t$ in $T$ belongs to some element of $\mathrm{B}$. We write $\mathrm{B}_{t}$ to denote the elements of the bundle $\mathrm{B}$ which contain the point $t$. Then, the bundle condition can be written as $\bigcup_{t \in T} \mathrm{~B}_{t}=T$. As mentioned above, the elements of $\mathrm{B}$ can be thought of as the admissible paths. A pair $\langle\mathrm{T}, \mathrm{B}\rangle$ in which $\mathrm{B}$ is a bundle on $\mathrm{T}$ is called a bundled tree. We say that $\langle\mathrm{T}, \mathrm{B}\rangle$ is a complete bundled tree when $\mathrm{B}=\mathrm{H}(\mathrm{T})$. 


\subsection{Classes of trees and bundled trees generated by linear orders}

Definition 3.1 With every class $\mathcal{C}$ of linear orders we associate the following classes of trees and bundled trees:

1. $\mathcal{T}(\mathcal{C})$ : the class of all trees in which every path is in $\mathcal{C}$.

2. $\mathcal{B}(\mathcal{C})$ : the class of all bundled trees $\langle\mathrm{T}, \mathrm{B}\rangle$ such that $\mathrm{T} \in \mathcal{T}(\mathcal{C})$.

3. $\mathcal{B}^{+}(\mathcal{C})$ : the class of all bundled trees $\langle\mathrm{T}, \mathrm{B}\rangle$ such that every path in the bundle $\mathrm{B}$ is in $\mathcal{C}$.

4. $\mathcal{T}^{*}(\mathcal{C})$ : the class of all trees $\mathrm{T}$ such that $\langle\mathrm{T}, \mathrm{B}\rangle$ belongs to $\mathcal{B}^{+}(\mathcal{C})$ for some bundle $\mathrm{B}$ on $\mathrm{T}$, i.e., $\mathrm{T}$ contains a bundle of paths from $\mathcal{C}$.

5. $\mathcal{B}^{*}(\mathcal{C})$ : the class of all bundled trees $\langle\mathrm{T}, \mathrm{B}\rangle$ such that $\mathrm{T}$ belongs to $\mathcal{T}^{*}(\mathcal{C})$

A tree in $\mathcal{T}(\alpha)$, where $\alpha$ is a linear order type, will be called a synchronizable tree because all paths in it, being isomorphic to each other, can be thought of as alternative copies of the same flow of time (see [6]).

In the above definitions of the classes $\mathcal{T}(\mathcal{C}), \mathcal{B}(\mathcal{C})$, etc., we have considered trees and bundled trees separately. For technical convenience and uniformity reasons, from now on we will often identify any tree $\mathrm{T}$ with the corresponding complete bundled tree $\langle\mathrm{T}, \mathrm{H}(\mathrm{T})\rangle$. This will give meaning to expressions like $\mathrm{T} \in \mathcal{B}(C)$.

Definition 3.2 The bundled tree $\langle\mathrm{T}, \mathrm{B}\rangle$ is contained in the bundled tree $\left\langle\mathrm{T}^{\prime}, \mathrm{B}^{\prime}\right\rangle$ whenever $\mathrm{T} \subseteq \mathrm{T}^{\prime}$ and $\mathrm{B} \subseteq \mathrm{B}^{\prime}$. We also say that $\langle\mathrm{T}, \mathrm{B}\rangle$ is a bundled subtree of $\left\langle\mathrm{T}^{\prime}, \mathrm{B}^{\prime}\right\rangle$.

Definition 3.3 Given a class of linear orders $\mathcal{C}$, a linear order $\mathrm{L}$ is a limit order for $\mathcal{C}$ if there exists a bundled tree $\langle\mathrm{T}, \mathrm{B}\rangle \in \mathcal{B}^{+}(\mathcal{C})$ such that $\mathrm{L} \in \mathrm{H}(\mathrm{T})$. In this case, we also say that the bundle $\mathrm{B}$ generates $\mathrm{L}$. If $\mathrm{L} \notin \mathrm{B}$ then $\mathrm{L}$ will be called an emergent path in $\mathrm{B}$. Likewise, if $\mathrm{L} \notin \mathcal{C}$, we say that $\mathrm{L}$ is an emergent order in $\mathcal{C}$.

The class of limit orders for $\mathcal{C}$ will be denoted by $\mathcal{C}^{*}$ and will be called the limit closure of $\mathcal{C}$. The class $\mathcal{C}$ is closed whenever it is equal to $\mathcal{C}^{*}$ 
Note that every linear order from $\mathcal{C}$ is trivially a limit order for $\mathcal{C}$, so that the inclusion $\mathcal{C} \subseteq \mathcal{C}^{*}$ always holds.

By the bundle condition that every element in a tree $\mathrm{T}$ belongs to some element of any bundle $\mathrm{B}$, so that $T=\bigcup \mathrm{B}$, we have the following characterization of emergent paths.

Proposition 3.4 The following conditions are equivalent:

1. The path $h$ emerges in the bundle $\mathrm{B}$;

2. $h \notin \mathrm{B}$ and for every $x \in h$ there exists a path $h_{x} \in \mathrm{B}$ such that $x \in h_{x} \cap h$;

3. $h \notin \mathrm{B}$ and $h$ is the union of proper initial segments of elements of $\mathrm{B}$.

For example, let $\mathcal{C}$ be the class $\mathcal{C}_{\text {fin }}$ of all finite linear orders. Then $\mathcal{B}^{+}(\mathcal{C})$ contains the bundled tree $\left\langle\mathrm{T}_{0}, \mathrm{~B}_{0}\right\rangle$ of Figure 1 , in which $\mathrm{B}_{0}$ consists of all finite paths $h_{1}, h_{2}, \ldots$ The path $h_{\omega}$ emerges in $\mathrm{B}_{0}$ and is isomorphic to the linear order of natural numbers. Thus, any linear order of order type $\omega$ is a limit order for $\mathcal{C}_{\text {fin }}$.

Figure 1

$$
\mathrm{T}_{0}
$$

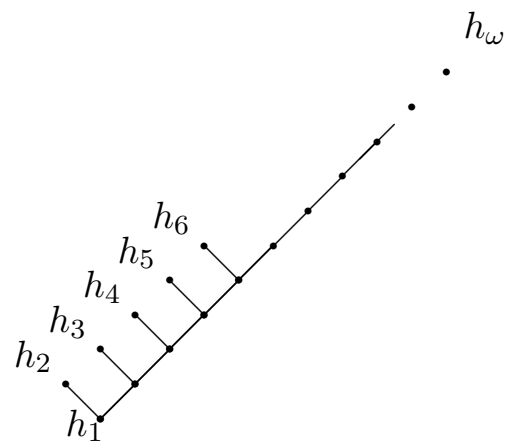


Proposition 3.5 For every limit linear order $\mathrm{L} \in \mathcal{C}^{*}$, there exists a bundled tree $\left\langle\mathrm{T}^{\mathrm{L}}, \mathrm{B}^{\mathrm{L}}\right\rangle$ in $\mathcal{B}^{+}(\mathcal{C})$ which generates $\mathrm{L}$ and has no emerging linear order different from L. A bundled tree with this property will be called a lean generating bundled tree for $\mathrm{L}$ in $\mathcal{C}$.

Note that there can be different (non-isomorphic) lean generating bundled trees for a given linear order.

Proof. If $\mathrm{L} \in \mathcal{C}$, then take $\mathrm{T}^{\mathrm{L}}$ to be just $\mathrm{L}$ and $\mathrm{B}^{\mathrm{L}}$ to be $\{\mathrm{L}\}$. In this case $\left\langle\mathrm{T}^{\mathrm{L}}, \mathrm{B}^{\mathrm{L}}\right\rangle$ has no emerging linear order.

If $\mathrm{L} \in \mathcal{C}^{*} \backslash \mathcal{C}$, we consider a bundled tree $\langle\mathrm{T}, \mathrm{B}\rangle \in \mathcal{B}^{+}(\mathcal{C})$ such that $\mathrm{L} \in \mathrm{H}(\mathrm{T})$. Let $\alpha$ be the cofinality type of $\mathrm{L}$. For $\beta<\alpha$, we write $x_{\beta}$ for the image of $\beta$ in a fixed cofinal embedding of $\alpha$ in L. For any $\beta<\alpha$, we let $h_{\beta}$ be any path in $\mathrm{B}$ such that $x_{\beta} \in \mathrm{L} \cap h_{\beta}$.

We define the bundles $\mathrm{B}_{\beta}(\beta \leq \alpha)$ by transfinite induction on $\alpha$. $\mathrm{B}_{0}$ is the singleton set $\left\{h_{0}\right\}$. For $\beta=\gamma+1$, we let $\mathrm{B}_{\beta}=\mathrm{B}_{\gamma}$ if $x_{\beta} \in h_{\gamma}$, and $\mathrm{B}_{\beta}=\mathrm{B}_{\gamma} \cup\left\{h_{\beta}\right\}$, otherwise. Finally, if $\beta$ is a limit ordinal, we set $\mathrm{B}_{\beta}=\bigcup_{\gamma<\beta} \mathrm{B}_{\gamma}$.

We let $\mathrm{B}^{\mathrm{L}}$ be $\mathrm{B}_{\alpha}$ and $T^{\mathrm{L}}$ be $\cup \mathrm{B}^{\mathrm{L}}$. The order $\prec^{\mathrm{L}}$ in $T^{\mathrm{L}}$ is the one induced by the orders on paths in $\mathrm{B}^{\mathrm{L}}$. Clearly, $\left\langle\mathrm{T}^{\mathrm{L}}, \mathrm{B}^{\mathrm{L}}\right\rangle \in \mathcal{B}^{+}(\mathcal{C})$ and $\mathrm{L} \in \mathrm{H}\left(\mathrm{T}^{\mathrm{L}}\right)$. Moreover, every point $x \notin \mathrm{L}$ in $\mathrm{T}^{\mathrm{L}}$ belongs to exactly one $h_{\beta}$ and hence $\mathrm{L}$ is the only emerging path in $\mathrm{B}_{\mathrm{L}}$.

Proposition 3.6 The limit closure of a class of linear orders closed under isomorphisms is a topological closure operator, i.e., satisfies the following properties:

1. $\mathcal{C} \subseteq \mathcal{C}^{*}$.

2. If $\mathcal{C}_{1} \subseteq \mathcal{C}_{2}$ then $\mathcal{C}_{1}^{*} \subseteq \mathcal{C}_{2}^{*}$.

3. $\mathcal{C}^{*}=\mathcal{C}^{* *}$

4. $\left(\mathcal{C}_{1} \cup \mathcal{C}_{2}\right)^{*}=\mathcal{C}_{1}^{*} \cup \mathcal{C}_{2}^{*}$.

Proof. Claims 1. and 2. are trivial. For 3, consider any linear order L in $\mathcal{C}^{* *}$. There exists a lean generating bundled tree $\left\langle\mathrm{T}^{\mathrm{L}}, \mathrm{B}^{\mathrm{L}}\right\rangle \in \mathcal{B}^{+}\left(\mathcal{C}^{*}\right)$ for $\mathrm{L}$ 
in $\mathcal{C}^{*}$ like in the proof of Proposition 3.5, i.e., such that: (1) for a suitable cardinal $\alpha, \mathrm{B}^{\mathrm{L}}=\left\{h_{\beta}^{*} \mid \beta<\alpha\right\} \subseteq \mathcal{C}^{*}$, (2) $T^{\mathrm{L}}=\bigcup \mathrm{B}^{\mathrm{L}}$, and $(3) \mathrm{L} \in \mathrm{H}\left(\mathrm{T}^{\mathrm{L}}\right)$.

Similarly, for every path $h_{\beta}^{*}$ there exists a lean generating bundled tree $\left\langle\mathrm{T}_{\beta}, \mathrm{B}_{\beta}\right\rangle \in \mathcal{B}^{+}(\mathcal{C})$ for $h_{\beta}^{*}$ in $\mathcal{C}$. This means in particular that, for every element $x$ of $h_{\beta}^{*}$, there is a path $h_{x} \in \mathrm{B}_{\beta}$ such that $x \in h_{x} \cap h_{\beta}^{*}$. Since the class $\mathcal{C}$ is closed under isomorphisms, we can assume that (1) for any $\beta$ and $x \in \mathrm{L}, h_{x} \cap \mathrm{L}$ is an initial segment of each of $h_{x}$ and $\mathrm{L}$ (and is contained in $h_{x} \cap h_{\beta}^{*}$ ), and (2) for $\beta \neq \beta^{\prime}$, the trees $\mathrm{T}_{\beta}$ and $\mathrm{T}_{\beta^{\prime}}$ intersect only on $\mathrm{L}$.

We now consider the bundled tree $\left\langle\mathrm{T}^{\prime}, \mathrm{B}^{\prime}\right\rangle$ in which $\mathrm{B}^{\prime}$ is $\bigcup_{\beta<\alpha} \mathrm{B}_{\beta}$ and $\mathrm{T}^{\prime}$ is $\cup^{\prime}$. This bundled tree is in $\mathcal{B}^{+}(\mathcal{C})$ and can be viewed as the result of replacing every $h_{\beta}^{*}$ in $\langle\mathrm{T}, \mathrm{B}\rangle$ by $\mathrm{B}_{\beta}$. Moreover, for every $x \in \mathrm{L}$, there is a $h_{x}^{\prime}$ in $\mathrm{B}^{\prime}$ such that $x \in h_{x}^{\prime} \cap \mathrm{L}$. Thus, $\left\langle\mathrm{T}^{\prime}, \mathrm{B}^{\prime}\right\rangle$ generates $\mathrm{L}$ and hence $\mathrm{L} \in \mathcal{C}^{*}$.

Finally, to prove 4 , first note that the set $\mathcal{C}_{1}^{*} \cup \mathcal{C}_{2}^{*}$ is trivially included in $\left(\mathcal{C}_{1} \cup \mathcal{C}_{2}\right)^{*}$. For the converse, assume $h^{*} \in\left(\mathcal{C}_{1} \cup \mathcal{C}_{2}\right)^{*}$. Then, there is a (lean) generating bundled tree $\langle\mathrm{T}, \mathrm{B}\rangle \in B^{+}\left(\mathcal{C}_{1} \cup \mathcal{C}_{2}\right)$ for $h^{*}$ in $\mathcal{C}_{1} \cup \mathcal{C}_{2}$. According to the proof of Proposition 3.5, for any $x$ in a cofinal subset $X$ of $h^{*}$, there is a path $h_{x} \in \mathcal{C}_{1} \cup \mathcal{C}_{2}$ such that $x \in h^{*} \cap h_{x}$. Call $X_{1}$ and $X_{2}$ the subsets of $X$ such that the path $h_{x}$ can be chosen in $\mathcal{C}_{1}$, respectively in $\mathcal{C}_{2}$. At least one of these two sets is cofinal in $h^{*}$ and, by the proof of Proposition 3.5 again, if $X_{i}$ is cofinal in $h^{*}$, then $h^{*} \in \mathcal{C}_{i}^{*}$.

The topology induced by this closure operator on all paths in a tree is essentially the one considered by Sabbadin and Zanardo in [13].

Some examples:

- The classes $\mathcal{C}_{\text {lin }}, \mathcal{C}_{\text {first }}, \mathcal{C}_{\text {discr }}, \mathcal{C}_{\text {dense }}, \mathcal{C}_{\text {Ded }}, \mathcal{C}_{\text {cont }}, \mathcal{C}_{\text {wf }},\{\omega\},\{\zeta\}$ are closed.

- The classes $\mathcal{C}_{\text {fin }}, \mathcal{C}_{\text {last }}, \mathcal{C}_{\text {iwf }},\{\eta\},\{\lambda\}$ are not closed. For the last two classes this claim is not so obvious and it will be proved below.

- $\mathcal{C}_{\text {fin }}^{*}=\mathcal{C}_{\text {fin }} \cup\{\omega\} ; \mathcal{C}_{\text {last }}^{*}=\mathcal{C}_{\text {lin }} ; \mathcal{C}_{\text {iwf }}^{*}=\mathcal{C}_{\text {iwf }} \cup\left(\mathcal{C}_{\text {iwf }}+\{\omega\}\right)$.

Definition 3.7 The long rational line is the linear order $\mathrm{L}_{\mathbb{Q}}=\langle X,<\rangle$ in which $X=\omega_{1} \cup \mathbb{Q}^{-} \cup \bigcup_{\alpha \in \omega_{1}} \mathbb{Q}_{\alpha}, \mathbb{Q}^{-}$and the $\mathbb{Q}_{\alpha}$ 's $\left(\alpha<\omega_{1}\right)$ are disjoint copies of $\mathbb{Q}$, and $<$ has the following properties:

(1) it agrees with the usual order on $\omega_{1}$ and on any copy of the rationals, 
(2) for every $\alpha \in \omega_{1}$ and $x \in \mathbb{Q}_{\alpha}, \alpha<x<\alpha+1$, and

(3) for every $x \in \mathbb{Q}^{-}, x<\emptyset$, the first element of $\omega_{1}$.

The long real line ${ }^{1}$ is defined similarly, by replacing $\mathbb{Q}$ with $\mathbb{R}$.

Roughly speaking, the long rational (real) line is obtained by inserting a copy of the rationals (reals) between any ordinal and its successor in $\omega_{1}$, and by preceding $\emptyset$ in $\omega_{1}$ by a copy of the rationals (reals). In the next proposition we will use the fact that any countable ordinal can be order embedded in $\mathbb{Q}$, and hence in $\mathbb{R}$, too (see [12]).

Proposition 3.8 The long rational (resp. real) line is an emerging path in $\{\eta\}$ (resp. $\{\zeta\})$, and hence these classes are not closed.

Proof. The two cases can be dealt with in the very same way; so we prove the proposition for the long rational line only. In particular, we show that, for every limit ordinal $\alpha \in \omega_{1}$, the set $\left\{x \in \mathrm{L}_{\mathbb{Q}}: x<\alpha\right\}$ is order isomorphic to $\mathbb{Q}$ (and hence to an initial segment of $\mathbb{Q}$ ), so that Proposition 3.4 can be used.

Consider an order embedding $f$ of $\alpha$ into $\mathbb{Q}$. The set $\{q \in Q: q<f(\emptyset)\}$ is isomorphic to $\mathbb{Q}$, as well as any set $\{q \in Q: f(\beta)<q<f(\beta+1)\}$, with $\beta<\alpha$. Then $\mathbb{Q}$ is isomorphic to $\left\{x \in \mathrm{L}_{\mathbb{Q}}: x<\alpha\right\} .^{2}$

To conclude that $\{\eta\}$ is not closed, we have only to observe that $\mathrm{L}_{\mathbb{Q}}$ is not isomorphic to $\eta$ because its cofinality type is $\omega_{1}$.

Lemma 3.9 Let $\mathrm{L}_{1}$ and $\mathrm{L}_{2}$ be linear orders such that, for every $x_{i} \in L_{i}$, the set $\left\{x \in L_{i}: x<_{i} x_{i}\right\}$ is order isomorphic to $\mathbb{Q}$ (resp. $\mathbb{R}$ ). Then:

1) the cofinality type of $\mathrm{L}_{1}$ and $\mathrm{L}_{2}$ is either $\omega$ or $\omega_{1}$, and

2) $\mathrm{L}_{1}$ and $\mathrm{L}_{2}$ are isomorphic if and only if they have the same cofinality type.

Proof. Again, both cases are analogous, and we will only consider the case of reals. Assume, for reductio ad absurdum, that the cofinality type

\footnotetext{
${ }^{1}$ A slightly different definition of the long real line is given in [8]

${ }^{2}$ In the case of $\mathrm{L}_{\mathbb{Q}}$, we could have simply observed that the set $\left\{x \in \mathrm{L}_{\mathbb{Q}}: x<\alpha\right\}$ is isomorphic to $\mathbb{Q}$ because it is a countable dense linear order with no first and no last element.
} 
of $\mathrm{L}_{1}$ is $\alpha>\omega_{1}$ and let $f$ be an order embedding of $\alpha$ into $\mathrm{L}_{1}$. By the assumptions on $\mathrm{L}_{1}$, the set $X=\left\{x \in \mathrm{L}_{1}: x<f\left(\omega_{1}\right)\right\}$ is order isomorphic to the reals, but the restriction of $f$ to $\omega_{1}$ is an order embedding of $\omega_{1}$ in $X$, which is impossible, because every initial segment of $\mathbb{R}$ has a cofinality $\omega$. This contradiction concludes the proof of the first claim.

If $\mathrm{L}_{1}$ and $\mathrm{L}_{2}$ have different cofinality type, they cannot be isomorphic. Assume now that $\alpha$ is the cofinality type of both $\mathrm{L}_{1}$ and $\mathrm{L}_{2}$. For $\beta<\alpha$ we denote by $x_{i}^{\beta}$ the image of $\beta$ in a cofinal embedding of $\alpha$ in $\mathrm{L}_{i}$, and by $X_{i}^{\beta}$ the set $\left\{x \in \mathrm{L}_{i}: x<_{i} x_{i}^{\beta}\right\}$. For any $\beta<\alpha$, we define an isomorphism $g^{\beta}$ from $X_{1}^{\beta}$ onto $X_{2}^{\beta}$ such that, for $\gamma<\beta, g^{\gamma} \subseteq g^{\beta}$.

Both sets $X_{1}^{0}$ and $X_{2}^{0}$ are isomorphic to $\mathbb{R}$ and hence we can fix an isomorphism $g^{0}$ from $X_{1}^{0}$ onto $X_{2}^{0}$. Assume that $g^{\beta}$ is defined and consider the sets $Y_{i}^{\beta}=X_{i}^{\beta+1} \backslash X_{i}^{\beta}$. Since both $X_{i}^{\beta+1}$ and $X_{i}^{\beta}$ are isomorphic to the reals, the sets $Y_{i}^{\beta}$ are isomorphic to the set of non-negative reals and hence there is an order isomorphism $g$ from $Y_{1}^{\beta}$ onto $Y_{2}^{\beta}$. We let $g^{\beta+1}$ be $g^{\beta} \cup g$. When $\beta$ is a limit ordinal, we let $g^{\beta}$ be $\bigcup_{\gamma<\beta} g^{\gamma}$.

It is readily verified that, for $\gamma<\beta<\alpha, g^{\gamma} \subseteq g^{\beta}$ and hence $\bigcup_{\beta<\alpha} g^{\beta}$ is an isomorphism of $\mathrm{L}_{1}$ onto $\mathrm{L}_{2}$.

On the basis of this lemma we have a precise description of the closure of the classes $\{\eta\}$ and $\{\zeta\}$ :

$$
\{\eta\}^{*}=\left\{\eta, \mathrm{L}_{\eta}\right\} \quad\{\zeta\}^{*}=\left\{\zeta, \mathrm{L}_{\zeta}\right\}
$$

where $\mathrm{L}_{\eta}$ and $\mathrm{L}_{\zeta}$ respectively denote the order types of the long rational line and of the long real line.

We can now extend Definition 3.1 and associate with any class of linear orders $\mathcal{C}$ the class of trees $\mathcal{T}\left(\mathcal{C}^{*}\right)$ and the class of bundled trees $\mathcal{B}\left(\mathcal{C}^{*}\right)$ obtained by applying the limit closure operator to $\mathcal{C}$.

Here are some relationships between the classes introduced above.

Proposition 3.10 For any class $\mathcal{C}$ of linear orders:
1. $\mathcal{T}(\mathcal{C}) \subseteq \mathcal{B}(\mathcal{C}) \subseteq \mathcal{B}^{+}(\mathcal{C}) \subseteq \mathcal{B}^{*}(\mathcal{C}) \subseteq \mathcal{B}\left(\mathcal{C}^{*}\right)=\mathcal{B}^{+}\left(\mathcal{C}^{*}\right)$
2. $\mathcal{T}(\mathcal{C}) \subseteq \mathcal{T}^{*}(\mathcal{C}) \subseteq \mathcal{T}\left(\mathcal{C}^{*}\right)=\mathcal{T}^{*}\left(\mathcal{C}^{*}\right) \subseteq \mathcal{B}\left(\mathcal{C}^{*}\right)$ 
3. For every closed class $\mathcal{C}$ :

$$
\mathcal{T}(\mathcal{C})=\mathcal{T}^{*}(\mathcal{C})=\mathcal{T}\left(\mathcal{C}^{*}\right) \text { and } \quad \mathcal{B}(\mathcal{C})=\mathcal{B}^{+}(\mathcal{C})=\mathcal{B}\left(\mathcal{C}^{*}\right)=\mathcal{B}^{*}(\mathcal{C})
$$

Proof. Most of these inclusions are immediate consequences from the definitions. For instance, to show $\mathcal{T}^{*}(\mathcal{C}) \subseteq \mathcal{T}\left(\mathcal{C}^{*}\right)$, consider any element $\mathrm{T}$ of $\mathcal{T}^{*}(\mathcal{C})$. This means that, for a suitable $\mathrm{B} \subseteq \mathcal{C},\langle\mathrm{T}, \mathrm{B}\rangle$ is a bundled tree $\left(\right.$ in $\mathcal{B}^{+}(\mathcal{C})$ ). For every path $h$ in $\mathrm{T}$ and any $x \in h$, by the bundle condition that any element of the tree belongs to some path in the bundle, there is an element $h_{x}$ of B such that $x \in h_{x}$. Since $h$ and $h_{x}$ are paths in the same tree, this implies $x \in h \cap h_{x}$. Thus, $h \in \mathcal{C}^{*}$ because $\mathrm{B} \subseteq \mathcal{C}$. Hence, $\mathrm{T} \in \mathcal{T}\left(\mathcal{C}^{*}\right)$.

Now, applying the inclusion above to $\mathcal{C}^{*}$ and using the closure properties from Proposition 3.6, we have $\mathcal{T}^{*}\left(\mathcal{C}^{*}\right) \subseteq \mathcal{T}\left(\mathcal{C}^{* *}\right)=\mathcal{T}\left(\mathcal{C}^{*}\right)$. The inclusion $\mathcal{T}\left(\mathcal{C}^{*}\right) \subseteq \mathcal{T}^{*}\left(\mathcal{C}^{*}\right)$ is obvious, hence $\mathcal{T}\left(\mathcal{C}^{*}\right)=\mathcal{T}^{*}\left(\mathcal{C}^{*}\right)$

In general, the classes $\mathcal{T}(C)$ and $\mathcal{T}^{*}(C)$ are different. For instance, if $C$ is the class of finite linear orders, then $\mathcal{T}^{*}(C)$ contains the tree $\mathrm{T}_{0}$ of Figure 3.1 with an emerging $\omega$-path.

Also, the inclusion $\mathcal{T}^{*}(\mathcal{C}) \subseteq \mathcal{T}\left(\mathcal{C}^{*}\right)$ is proper whenever $\mathcal{C}$ is not closed. If, in fact, $h$ is an emerging path in $\mathcal{C}$, then the tree consisting of just $h$ is a tree in $\mathcal{T}\left(\mathcal{C}^{*}\right) \backslash \mathcal{T}^{*}(\mathcal{C})$. In general, a tree $\mathrm{T}$ in $\mathcal{T}\left(\mathcal{C}^{*}\right)$ is not in $\mathcal{T}^{*}(\mathcal{C})$ if some path $h$ in $\mathrm{T}$ is an emerging path in $\mathcal{C}$, but no bundle $\mathrm{B} \subseteq \mathcal{C}$ in $\mathrm{T}$ generates $h$. Thus, if we extend $\mathrm{T}$ with suitable new paths, we can obtain a new tree from $\mathcal{T}\left(\mathcal{C}^{*}\right)$, in which $h$ is generated by a bundle. More precisely, $h$ turns out to have this property if $\mathrm{T}$ is extended with the bundle of a lean generating bundled tree for $h$ in $\mathcal{C}$. The procedure can be applied to every emerging path and a tree in $\mathcal{T}^{*}(\mathcal{C})$ is eventually obtained. This proves the following proposition.

Proposition 3.11 Every tree from $\mathcal{T}\left(\mathcal{C}^{*}\right)$ is contained in a tree from $\mathcal{T}^{*}(\mathcal{C})$.

Given any class $\mathcal{C}$ of linear orders, we put

$$
\mathbb{T}(\mathcal{C})=\left\{\mathcal{T}(\mathcal{C}), \mathcal{B}(\mathcal{C}), \mathcal{B}^{+}(\mathcal{C}), \mathcal{T}^{*}(\mathcal{C}), \mathcal{B}^{*}(\mathcal{C}), \mathcal{T}\left(\mathcal{C}^{*}\right), \mathcal{B}\left(\mathcal{C}^{*}\right)\right\}
$$

Proposition 3.10 above shows that $\mathbb{T}(\mathcal{C})$ cannot be extended by further closure operations starting with $\mathcal{C}$. 


\section{On the branching time logics over classes of trees associated with a class of linear orders}

\subsection{Linear and branching time temporal logics}

The basic Priorean propositional language for linear time temporal logics consists of the usual Boolean part plus the temporal operators $P$ and $F$ (with dual operators $H=\neg P \neg$ and $G=\neg F \neg$ ). The semantics of these operators is the usual Kripke semantics for modal logics. Given any linear order $\mathrm{L}=\langle X,<\rangle$ and any evaluation $V$ of the propositional variables in $X$, truth of Boolean combinations of formulae in the model $M=\langle\mathrm{L}, V\rangle$ is defined in the usual way, while for temporal operators we put:

$$
\begin{array}{lll}
M, t \vDash F \theta & \text { iff } & M, t^{\prime} \vDash \theta, \text { for some } t^{\prime}>t \\
M, t \vDash P \theta & \text { iff } & M, t^{\prime} \vDash \theta, \text { for some } t^{\prime}<t
\end{array} .
$$

Given a Kripke model $M=\langle\mathrm{T}, V\rangle$ based on a tree $\mathrm{T}$, a path $h$ in $\mathrm{T}$ and a moment $t \in h$, the truth of a $P F$-temporal formula $\theta$ in $M$ at $\langle h, t\rangle$, denoted $M, h, t \vDash \theta$, is defined as truth of $\theta$ at the point $t$ in the linear order $\left\langle h, \prec_{\mid h}\right\rangle$, with the evaluation $V^{\prime}=V_{\mid h}$.

In a branching time context, the modality $\diamond$ is read as 'for some possible path' passing through the current moment. Thus, we can put

$$
M, h, t \vDash \diamond \theta \quad \Leftrightarrow \quad M, h^{\prime}, t \vDash \theta, \text { for some } h^{\prime} \in \mathrm{H}_{t}(\mathrm{~T})
$$

As usual, we write $\square$ for the dual operator $\neg \diamond \neg$. The respective truth condition for $\square$ is:

$$
M, h, t \vDash \square \theta \quad \Leftrightarrow \quad M, h^{\prime}, t \vDash \theta, \text { for every } h^{\prime} \in \mathrm{H}_{t}(\mathrm{~T})
$$

Note that these truth conditions do not depend on the path $h$.

This semantics can be seamlessly generalized to bundled trees, where 'for some possible path' means 'for some possible path from the bundle'. Formally, in the bundled tree interpretation of $\diamond$, the quantification over all paths in the tree on the right part of (4.3) is replaced by a quantification over B:

$$
M, h, t \vDash \diamond \theta \quad \Leftrightarrow \quad M, h^{\prime}, t \vDash \theta, \text { for some } h^{\prime} \in \mathrm{B}_{t}
$$


where $\mathrm{B}_{t}$ is the set of paths from B passing through $t$.

\subsection{A unified approach to logics for branching time}

By suitably combining the temporal operators $F, P$ with the modality $\diamond$ we can obtain a hierarchy of temporal logics for branching time studied in the literature - see e.g. [11], [4], [5], [2]. Here are some natural combinations of tenses and modalities, and the resulting languages when these are added to propositional language:

- $\diamond F$ : the basic Priorean modal language $\mathcal{L}_{\text {Prior }}^{m}$;

- $\diamond F, \diamond P$ : the basic Priorean temporal language $\mathcal{L}_{\text {Prior }}^{t}$;

- $\diamond F, \square F$ : the future fragment of the Peircean language $\mathcal{L}_{\text {Peirce; }}^{m}$;

- $\diamond F, \square F, \diamond P, \square P$ : the full Peircean language $\mathcal{L}_{\text {Peirce; }}$

- $\diamond, F$ : the future fragment of the Ockhamist language $\mathcal{L}_{\text {Ockham }}^{m}$;

- $\diamond, F, P$ : the full Ockhamist language $\mathcal{L}_{\text {Ockham }}$.

The semantical rules for $\mathcal{L}_{\text {Ockham }}$ in the tree or bundled tree semantics are given by (4.2), (4.3) and (4.5), in addition to the usual Boolean semantics. These rules accordingly provide semantics for all fragments of $\mathcal{L}_{\text {Ockham }}$ listed above, as well. Thus, we only consider one semantics for branching time logics over trees, viz. the Ockhamist semantics, of which the Priorean and Peircean semantics are now regarded as restrictions over the respective fragments. Nevertheless, in order to indicate explicitly which fragment we are considering, we will sometimes index the truth relation accordingly as $\vDash_{\text {Prior }}, \vDash_{\text {Peirce }}$, and $\vDash_{\text {Ockham }}$, and will use $\vDash_{\text {lin }}$ for the standard (Priorean) semantics over linear orders.

Given a class $\mathcal{T}$ of trees, the logics over $\mathcal{T}$ of the various languages listed above will be denoted as $L_{\text {Prior }}^{m}(\mathcal{T}), L_{\text {Prior }}^{t}(\mathcal{T})$, and $L_{\text {Prior }}(\mathcal{T})$ when referring to either of these. Likewise for the Peircean and Ockhamist logics. 
Note that tenses and modalities in the Priorean and Peircean logics occur only as part of composed operators. We will adopt the following notation:

$$
\begin{array}{llll}
\mathbf{F}:=\square F & \mathbf{f}:=\diamond F & \mathbf{G}:=\neg \mathbf{f} \neg & \mathbf{g}:=\neg \mathbf{F} \neg \\
\mathbf{P}:=\square P & \mathbf{p}:=\diamond P & \mathbf{H}:=\neg \mathbf{p} \neg & \mathbf{h}:=\neg \mathbf{P} \neg
\end{array}
$$

According to (4.2) and (4.3), given any branching time model $M=\langle\mathrm{T}, V\rangle$ and any moment $t$ in $\mathrm{T}$, the truth conditions for $\mathbf{F}$ and $\mathbf{f}$ are

$$
\begin{aligned}
& M, t \vDash \mathbf{F} \theta \quad \Leftrightarrow \quad \forall h \in \mathrm{H}_{t} \exists t^{\prime} \in h: t \prec t^{\prime} \text { and } M, t^{\prime} \vDash \theta \\
& M, t \vDash \mathbf{f} \theta \quad \Leftrightarrow \quad \exists h \in \mathrm{H}_{t} \exists t^{\prime} \in h: t \prec t^{\prime} \text { and } M, t^{\prime} \vDash \theta
\end{aligned}
$$

where the quantification over $\mathrm{H}_{t}$ is replaced by a quantification over $\mathrm{B}_{t}$ if bundled trees are considered. The truth conditions for $\mathbf{P}$ and $\mathbf{p}$ can be expressed similarly, by replacing $\prec$ with $\succ$. It should be observed, however, that the left-linearity property of trees implies that the interpretations of $\mathbf{P}$ and $\mathbf{p}$ coincide. These two operators actually agree with the linear time operator $P$. For this reason, in the sequel we will use only $\mathbf{P}$ and $\mathbf{H}$. The semantics for these operators is simply given by

$$
M, t \vDash \mathbf{P} \theta \quad \Leftrightarrow \quad \exists t^{\prime} \prec t: M, t^{\prime} \vDash \theta
$$

We have already observed that the truth of $\diamond \theta$ at $\langle t, h\rangle$ in $M$ does not depend on the path $h$ and hence the left-hand side of (4.3) is often written as $M, t \vDash \diamond \theta$. Likewise for $\square$. Formulae of this kind are called state formulae; more precisely, state formulae are those in which every temporal operator is in the scope of a $\diamond$, and hence their truth in a model only depends on the current moment, but not on the current path. Formulae in which every temporal operator is immediately prefixed by $\diamond$ or $\square$ will be called essentially state formulae.

Note that the Peircean (modal or temporal) language consists precisely of all essentially state formulae in the respective Ockhamist language.

Important examples of temporal logics with branching time semantics are the computation tree logic $\mathrm{CTL}^{*}$, which is the Ockhamist logic over the class of trees $\mathrm{T}(\omega)$, and its Peircean fragment CTL (see e.g. [7, 15]). 
Finally, it is also important to observe that, in the truth condition for $\mathbf{f}$ in (4.6), the double quantification over paths and points is equivalent to a quantification over points only:

$$
M, t \vDash \mathbf{f} \theta \quad \Leftrightarrow \quad \exists t^{\prime}: t \prec t^{\prime} \text { and } M, t^{\prime} \vDash \theta
$$

Likewise, for $\mathbf{G}$.

Thus, the Priorean language for branching time logics consists of those essentially state formulae in which the path quantification is trivialized.

\subsection{The variety of semantics over classes of trees}

In this section we investigate and compare the semantics determined by each of the 7 classes of trees associated with a given class of linear orders $\mathcal{C}$, defined earlier.

\subsubsection{On Priorean formulae}

First, we note that all classes of linear orders listed in Section 2 are definable in the Priorean temporal language (see $[14,9]$ ).

As noted above, no quantification over paths is essentially involved in the semantics for $\mathcal{L}_{\text {Prior }}$ and hence, for any class $\mathcal{C}$ of linear orders, $L_{\text {Prior }}(\mathcal{T}(\mathcal{C}))=$ $L_{\text {Prior }}(\mathcal{B}(\mathcal{C}))$.

As trivial consequences we obtain the equalities $L_{\text {Prior }}^{m}(\mathcal{T}(\mathcal{C}))=L_{\text {Prior }}^{m}(\mathcal{B}(\mathcal{C}))$ and $L_{\text {Prior }}^{t}(\mathcal{T}(\mathcal{C}))=L_{\text {Prior }}^{t}(\mathcal{B}(\mathcal{C}))$. Moreover, $L_{\text {Prior }}\left(\mathcal{B}^{+}(\mathcal{C})\right)=L_{\text {Prior }}\left(\mathcal{T}^{*}(\mathcal{C})\right)=$ $L_{\text {Prior }}\left(\mathcal{B}^{*}(\mathcal{C})\right)$.

Thus, for any Priorean formula $\theta$ and any tree $\mathrm{T}$ : $\mathrm{T} \vDash \theta$ iff $\langle\mathrm{T}, \mathrm{B}\rangle \vDash \theta$ for some bundled tree $\langle\mathrm{T}, \mathrm{B}\rangle$ iff $\langle\mathrm{T}, \mathrm{B}\rangle \vDash \theta$ for every bundled tree $\langle\mathrm{T}, \mathrm{B}\rangle$, i.e., the bundle does not play any role for the validity of Priorean formulae. Therefore, for every class of linear orders $\mathcal{C}$, the validities in $\mathcal{T}(\mathcal{C})$ and $\mathcal{B}(\mathcal{C})$ on the Priorean fragment trivially coincide. Likewise for the validities in $\mathcal{B}\left(\mathcal{C}^{*}\right)$ and $\mathcal{T}\left(\mathcal{C}^{*}\right)$, as well as the validities on $\mathcal{B}^{+}(\mathcal{C}), \mathcal{T}^{*}(\mathcal{C})$, and $\mathcal{B}^{*}(\mathcal{C})$.

On the other hand, note that the Priorean validities on $\mathcal{T}(\mathcal{C})$ and $\mathcal{T}\left(\mathcal{C}^{*}\right)$ in general differ, e.g. when $\mathcal{C}=\mathcal{C}_{\text {fin }}$. Likewise, the Priorean validities on $\mathcal{T}(\mathcal{C})$ and $\mathcal{B}^{+}(\mathcal{C})$ can differ, e.g.: $\mathcal{T}\left(\mathcal{C}_{\text {fin }}\right) \vDash G(G p \rightarrow p) \rightarrow G p$, while $\mathcal{B}^{+}\left(\mathcal{C}_{\text {fin }}\right) \not \models$ $G(G p \rightarrow p) \rightarrow G p$, witness the complete bundled tree over the tree $\mathrm{T}_{0}$ from 
Figure 3.1 with one $\omega$-path emerging from a set of finite paths. Finally, $\omega \not \models F G \perp$, while $\mathcal{B}^{+}\left(\mathcal{C}_{\text {fin }}\right) \vDash F G \perp$, hence the Priorean validities on $\mathcal{T}\left(\mathcal{C}^{*}\right)$ and on $\mathcal{B}^{+}(\mathcal{C})$ can differ.

Thus, in summary: the 7 classes of trees in $\mathbb{T}(\mathcal{C})$ determine 3 different cases of validities on each of the modal and temporal fragments of Prior, the strongest being $\vDash_{\mathcal{T}\left(\mathcal{C}^{*}\right)}$, which implies $\vDash_{\mathcal{T}^{*}(\mathcal{C})}$, which in turn implies $\vDash_{\mathcal{T}(\mathcal{C})}$.

\subsubsection{On Peircean formulae}

First, let us note that the Peircean validities on the class of all complete trees $\mathcal{T}\left(\mathcal{C}_{\text {lin }}\right)$, and on the class of all bundled trees $\mathcal{B}\left(\mathcal{C}_{\text {lin }}\right)$ coincide [5]. Still, we will show further that the modal Peircean fragment can distinguish these validities for some particular classes of linear orders, e.g. $\{\omega\}$. In fact, we will show that on the modal Peircean fragment, all 7 classes of trees from $\mathbb{T}(\mathcal{C})$ can produce different semantics. For that, taking into account the results of the previous subsection, and that any Priorean formula is also a Peircean formula, it will suffice to distinguish by means of Peircean formulae $\vDash_{\mathcal{T}(\mathcal{C})}$ from $\vDash_{\mathcal{B}(\mathcal{C})} ; \vDash_{\mathcal{T}\left(\mathcal{C}^{*}\right)}$ from $\vDash_{\mathcal{B}\left(\mathcal{C}^{*}\right)}$; and each of $\vDash_{\mathcal{T}^{*}(\mathcal{C})}, \vDash_{\mathcal{B}^{*}(\mathcal{C})}$, and $\vDash_{\mathcal{B}^{+}(\mathcal{C})}$ from the others.

1. $\mathcal{T}(\omega) \vDash \mathbf{G}(p \rightarrow \mathbf{f} p) \wedge \mathbf{f} p \rightarrow \mathbf{g f} p$ while $\mathcal{B}(\omega) \not \models \mathbf{G}(p \rightarrow \mathbf{f} p) \wedge \mathbf{f} p \rightarrow \mathbf{g f} p$ because this formula fails on the bundled binary $\omega$-tree where exactly one path is removed and $p$ is true precisely at all moments of that path.

Thus, in general $\vDash_{\mathcal{T}(\mathcal{C})} \neq \vDash_{\mathcal{B}(\mathcal{C})}$.

2. Since $\{\omega\}$ is closed, we thus also obtain that $\vDash_{\mathcal{T}\left(\mathcal{C}^{*}\right)} \neq \vDash_{\mathcal{B}\left(\mathcal{C}^{*}\right)}$.

3. Recall, that $\mathcal{B}^{+}(\mathcal{C}) \subseteq \mathcal{B}^{*}(\mathcal{C})$ and $\mathcal{T}^{*}(\mathcal{C}) \subseteq \mathcal{B}^{*}(\mathcal{C})$, hence $\vDash_{\mathcal{B}^{*}(\mathcal{C})}$ implies each of $\vDash_{\mathcal{B}^{+}(\mathcal{C})}$ and $\vDash_{\mathcal{T}^{*}(\mathcal{C})}$.

4. On the other hand, $\mathcal{B}^{+}\left(\mathcal{C}_{\text {fin }}\right) \vDash \mathbf{F G} \perp$ while $\mathcal{T}^{*}\left(\mathcal{C}_{\text {fin }}\right) \not \models \mathbf{F G} \perp$, and hence $\mathcal{B}^{*}\left(\mathcal{C}_{\text {fin }}\right) \not \models \mathbf{F G} \perp$. Thus, $\vDash_{\mathcal{B}^{+}(\mathcal{C})} \neq \vDash_{\mathcal{T}^{*}(\mathcal{C})}$ and $\vDash_{\mathcal{B}^{+}(\mathcal{C})} \neq \vDash_{\mathcal{B}^{*}(\mathcal{C})}$.

5. Finally, since $\mathcal{T}^{*}(\omega)=\mathcal{T}\left(\{\omega\}^{*}\right)=\mathcal{T}(\omega)$, and $\mathcal{B}^{*}(\omega)=\mathcal{B}\left(\{\omega\}^{*}\right)=\mathcal{B}(\omega)$, we have $\vDash_{\mathcal{T}^{*}(\mathcal{C})} \neq \vDash_{\mathcal{B}^{*}(\mathcal{C})}$. 


\subsubsection{On Ockhamist formulae}

As a consequence of the results of the previous subsection, we have that the 7 classes of trees from $\mathbb{T}(\mathcal{C})$ can also produce different Ockhamist semantics because Peircean language is a fragment of the the full Ockhamist language. This language, though, is rather more expressive on trees than its Peircean fragment.

For instance, the Ockhamist formula $\square G \diamond F p \rightarrow \diamond G F p([4])$ is valid in all complete trees, but can be falsified in some bundled tree, and hence, unlike the Peircean case, we have $\vDash_{\mathcal{T}\left(\mathcal{C}_{\text {lin }}\right)} \neq \vDash_{\mathcal{B}\left(\mathcal{C}_{\text {lin }}\right)}$ in the Ockhamist language. As an example, consider the binary $\omega$-tree $\mathrm{T}_{\omega}^{2}$. Any node in this tree can be labeled by 0 if it is the 'left successor' of the previous node, or by 1 if it is the 'right successor'; then, the paths in this tree correspond to denumerable sequences of 0 and 1 . Let $\mathrm{B}$ be the bundle on $\mathrm{T}_{\omega}^{2}$ consisting of all sequences with finitely many 1's and let $V(p)$ be the set of all nodes labeled with 1 . It is readily verified that, with this evaluation, the formulas $\square G \diamond F p$ and $\diamond G F p$ are respectively true and false at any node of the bundled tree $\left\langle\mathrm{T}_{\omega}^{2}, \mathrm{~B}\right\rangle$.

\section{Translations}

\subsection{Standard translation}

There is a well-known (see e.g. [1, Ch. II.2]) translation of the Priorean temporal logic to first-order logic (regarding truth and validity in Kripke models) and universal monadic second-order logic (regarding truth and validity in frames).

This translation accordingly extends to the Ockhamist language into the enrichment with path quantification of first-order logic over trees. The formula $\operatorname{ST}_{\text {Ockham }}(\varphi)[x, h]$, expressing the truth of an Ockhamist formula $\varphi$ with respect to a state in a given tree, denoted by the state variable $x$, and a path in the tree through that state, denoted by the path variable $h$, as follows:

- $\operatorname{ST}_{\text {Ockham }}\left(p_{i}\right)[x, h]:=P_{i}(x)$, where $P_{i}$ is a unary predicate associated with the variable $p_{i}$;

- $\operatorname{ST}_{\text {Ockham }}(\neg \varphi)[x, h]:=\neg \mathrm{ST}_{\text {Ockham }}(\varphi)[x, h]$; 
- $\operatorname{ST}_{\text {Ockham }}(\varphi \wedge \psi)[x, h]:=\operatorname{ST}_{\text {Ockham }}(\varphi)[x, h] \wedge \operatorname{ST}_{\text {Ockham }}(\psi)[x, h]$;

- $\mathrm{ST}_{\text {Ockham }}(F \varphi)[x, h]:=\exists x^{\prime}\left(x<x^{\prime} \wedge h\left(x^{\prime}\right) \wedge \mathrm{ST}_{\text {Ockham }}(\varphi)\left[x^{\prime} / x, h\right]\right)$, where $x^{\prime}$ is (the first) state variable not occurring in $\operatorname{ST}_{\text {Ockham }}(\varphi)[x, h]$;

- $\operatorname{ST}_{\text {Ockham }}(P \varphi)[x, h]:=\exists x^{\prime}\left(x^{\prime}<x \wedge \operatorname{ST}_{\text {Ockham }}(\varphi)\left[x^{\prime} / x, h\right]\right)$, where $x^{\prime}$ is (the first) state variable not occurring in $\operatorname{ST}_{\text {Ockham }}(\varphi)[x, h]$;

(Note that, by the properties of trees, the path reference becomes redundant in this case.)

- $\mathrm{ST}_{\text {Ockham }}(\diamond \varphi)[x, h]:=\exists h^{\prime}\left(h^{\prime}(x) \wedge \operatorname{ST}_{\text {Ockham }}(\varphi)\left[x, h^{\prime} / h\right]\right)$, where $h^{\prime}$ is (the first) path variable not occurring in $\operatorname{ST}_{\text {Ockham }}(\varphi)[x, h]$.

Eventually, validity of an Ockhamist formula $\varphi$ in a tree $\mathrm{T}$ is expressed as follows:

$$
\mathrm{T} \vDash_{\text {Ockham }} \varphi \quad \text { iff } \quad \mathrm{T} \vDash \forall \bar{P} \forall h \forall x\left(h(x) \rightarrow \mathrm{ST}_{\text {Ockham }}(\varphi)[x, h]\right) .
$$

The translation above is readily generalized to bundled trees by relativization of the path quantification to the bundle.

The translations for the Peircean and Priorean logics are obtained from the above by restricting to the respective fragments. We note that the path quantification in the translation of Priorean formulae becomes redundant and can be omitted.

\subsection{Syntactic translations preserving equivalence be- tween validity in linear orders and trees.}

Here we investigate the question: how to translate the Priorean $P F$-language for linear orders to the languages of Priorean, Peircean, and Ockhamist logics on trees in order to best preserve the meaning of the temporal formulae in the transition from linear orders to trees.

In the case of Priorean logic, an obvious translation is the embedding given by $F \mapsto \mathbf{f}$ and $P \mapsto \mathbf{P}$. This embedding can be seen as a particular case of translation to Peircean language, which will be considered below. For Ockhamist logic there is only one reasonable choice, too, namely the identical 
embedding of the linear time operators. It is obvious that a $P F$-formula is valid in a class $\mathcal{C}$ of linear orders if and only it it is Ockham valid in the class of bundled trees $\langle\mathrm{T}, \mathrm{B}\rangle$ in which $\mathrm{B} \subseteq \mathcal{C}$.

The situation is more interesting with Peircean logic where there are many possible choices. In principle, each occurrence of $F$ in a linear time formula $\theta$ can be translated either to $\mathbf{F}$ or to $\mathbf{f}$, and hence, if $\theta$ has $n$ occurrences of $F$, then there are $2^{n}$ possible ways of translating it to a Peircean formula.

Definition 5.1 A translation $\tau(\theta)$ of a Priorean formula $\theta$ into the Peircean language is syntactic, if it is obtained from $\theta$ by converting each occurrence of $P$ to $\mathbf{P}$ and each occurrence of $F$ to $\mathbf{f}$ or $\mathbf{F}$.

In general, arbitrary syntactic translations of linear time formulae to Peircean formulae do not faithfully transfer the meaning of formulae from linear orders to trees; such translations need not even preserve validity. A notable example is the formula

$$
F p \wedge F q \rightarrow F(p \wedge F q) \vee F(q \wedge F p) \vee F(p \wedge q)
$$

which says that time is linear towards the future. Both natural translations, $F \mapsto \mathbf{F}$ and $F \mapsto \mathbf{f}$, transform this formula into non-valid Peircean formulae. On the other hand, linearity towards the future can also be expressed by the formula

$$
F p \rightarrow G(p \vee F p \vee P p)
$$

and the translation $\mathbf{F} p \rightarrow \mathbf{G}(p \vee \mathbf{F} p \vee \mathbf{P} p)$ of this formula is Peirce valid, as well as the translation $\mathbf{f} p \rightarrow \mathbf{g}(p \vee \mathbf{f} p \vee \mathbf{P} p)$.

Example 5.2 The density axiom $F p \rightarrow F F p$ has eight different syntactic translations, but only three of them, namely, $\mathbf{f} p \rightarrow \mathbf{f f} p, \mathbf{F} p \rightarrow \mathbf{F f} p$, and $\mathbf{F} p \rightarrow \mathrm{ff} p$ are valid on dense trees, that is on trees in which all paths are dense. The validity of these formulae is easily checked, as well as the satisfiability (in dense trees) of the negation of $\mathbf{f} p \rightarrow \mathbf{F F} p, \mathbf{f} p \rightarrow \mathbf{F f} p$, and $\mathbf{f} p \rightarrow \mathbf{f F} p$. A particular construction is needed for dealing with the remaining two implications $\mathbf{F} p \rightarrow \mathbf{F F} p$ and $\mathbf{F} p \rightarrow \mathbf{f F} p$. 
Consider the dense tree $\mathrm{T}$ consisting of a main path $h_{0}=\mathbb{Q}^{+} \cup\{0\}$, the set of non-negative rational numbers, and of other paths $h_{x}\left(x \in \mathbb{Q}^{+}\right)$ such that: 1) every $h_{x}$ is isomorphic to $h_{0}$, 2) $h_{x} \cap h_{0}=[0, x]$, and 3) the paths $h_{x}$ intersect each other only on $h_{0}$ (which implies that, for $0<y<x$, $\left.h_{y} \cap h_{x}=[0, y]\right)$. This means that each point $x>0$ of $h_{0}$ is the initial point of a new branch and that the elements of that branch belong only to $h_{x}$.

Let $V$ be any evaluation such that $V(p)=h_{0}$. Then $\mathrm{T}, V, 0 \models \mathbf{F} p$ because every $h_{x}$ contains elements different from 0 of $h_{0}$. On the other hand, for every $x>0$ in $h_{0}$, we have $\mathrm{T}, V, x \models \mathbf{g} \neg p$ and hence $\mathrm{T}, V, 0 \models \mathbf{g g} \neg p$. This shows that $\mathbf{F} p \rightarrow \mathbf{F F} p$ is not a Peircean validity in the class of dense trees. To prove the same result for the formula $\mathbf{F} p \rightarrow \mathbf{f F} p$, we can use the same model and observe that $\mathbf{g} \neg p$ is true at any point different from the root and hence $\mathrm{T}, V, 0 \models \mathbf{G g} \neg p$.

As far as definability is concerned, it is straightforward to check that the formulae $\mathbf{f} p \rightarrow \operatorname{ff} p$ and $\mathbf{F} p \rightarrow \mathbf{F f} p$ can be falsified in any non-dense tree, and hence they define density in trees. The following construction shows, instead, a non-dense tree in which $\mathbf{F} p \rightarrow \mathrm{ff} p$ cannot be falsified, so that this formula, which is valid in dense trees, does not define this class. Let $\mathrm{T}$ be a tree with exactly two paths, $h_{1}$ and $h_{2}$, such that 1) $h_{1} \cap h_{2}$ is a singleton set, 2) $h_{1}$ is isomorphic to the non-negative rationals, and 3) $h_{2}$ is isomorphic to the non-negative rationals deprived of the open segment $(0,1)$, so that 1 is the immediate successor of 0 in $h_{2}$ and $\mathrm{T}$ is not dense. It is easily checked that $\mathbf{F} p \rightarrow \mathbf{f f} p$ cannot be falsified in $\mathrm{T}$.

Thus, a natural question arises to determine syntactic conditions under which a translation preserves validity, and, moreover, accordingly transfer the meaning. Two limit translations can be considered, for instance.

Definition 5.3 A Priorean formula is in a negation normal form if it is obtained from literals (propositional variables and their negations) by applying $\wedge, \vee, F, G, P, H$. Likewise, a Pericean formula is in negation normal form if is obtained from literals (prop. variables and their negations) by applying $\wedge, \vee, \mathbf{f}, \mathbf{g}, \mathbf{F}, \mathbf{G}, P, H$.

Definition 5.4 A Priorean formula $\theta$ is in modal disjunctive normal form if: 
- $\theta$ is a propositional formula, or

- $\theta=\bigvee \theta_{i}$ such that each $\theta_{i}$ is of the type $\alpha \wedge H \psi \wedge G \varphi \wedge P \psi_{1} \wedge . . \wedge P \psi_{k} \wedge$ $F \varphi_{1} \wedge \ldots \wedge F \varphi_{m}$ where $\alpha$ is a propositional formula (possibly just $\top$ or $\perp)$ and each $\psi, \psi_{1}, \ldots, \psi_{k}, \varphi, \varphi_{1}, \ldots, \varphi_{m}$ is in modal disjunctive normal form.

Since $\left(H \psi_{1} \wedge H \psi_{2}\right) \leftrightarrow H\left(\psi_{1} \wedge \psi_{2}\right)$ and $\left(G \varphi_{1} \wedge G \varphi_{2}\right) \leftrightarrow G\left(\varphi_{1} \wedge \varphi_{2}\right)$ are Priorean validities, every Priorean formula has a modal disjunctive normal form.

Definition 5.5 Let $\theta$ be a Priorean formula in modal disjunctive normal form.

1. The weakest translation $\tau^{w}(\theta)$ of $\theta$ to Peircean language is the result of replacing the occurrence of $P, H, F$, and $G$ in $\theta$ by, respectively, $\mathbf{P}, \mathbf{H}, \mathbf{f}$ and $\mathbf{g}$.

2. The strongest translation $\tau^{s}(\theta)$ is the result of replacing the occurrences of $P, H, F$, and $G$ in $\theta$ by, respectively, $\mathbf{P}, \mathbf{H}, \mathbf{F}$, and $\mathbf{G}$.

In general, by the weakest (respectively, strongest) translation of an arbitrary formula $\theta$, we mean the weakest (respectively, strongest) translation of any modal disjunctive normal form of $\theta$. It is routine to check that any two weakest (respectively, strongest) translations of a given formula are equivalent to each other. These translations will be respectively denoted by $\theta^{w}$ and $\theta^{s}$.

Note that if the Priorean formula $\theta$ is written using only $P$ and $F$ as primitive symbols, then $\theta^{w}$ is equivalent to the result of replacing $P$ by $\mathbf{P}$, every positive occurrence of $F$ by $\mathbf{f}$, and every negative occurrence of $F$ by F. The strongest translation of $\theta$ can be defined in the obviously opposite way.

Also, note that for every syntactic translation $\tau, \mathcal{T}(\mathcal{C}) \vDash_{\text {Peirce }} \tau(\theta)$ implies $\mathcal{C} \vDash_{\text {lin }} \theta$ because $\mathcal{C} \subseteq \mathcal{T}(\mathcal{C})$ and $\tau(\theta)$ is equivalent to $\theta$ on linear orders.

Every point-wise valuation $V$ (assigning a set of states to every propositional variable) over a tree $\mathcal{T}$, restricted to any $h \in \mathrm{H}(\mathrm{T})$ determines a valuation $V_{h}$ on that linear order. 
Thus, for any Priorean formula $\theta$, the Ockhamist formula $\diamond \theta$ satisfies the following:

$$
\mathrm{T}, V, t \vDash \diamond \theta \quad \text { iff } \quad \exists h \in \mathrm{H}_{t}(\mathrm{~T}): h, V_{h}, t \vDash_{\text {lin }} \theta
$$

and similarly for $\mathrm{T}, V, t \vDash \square \theta$. We thus have that, for every linear time formula $\theta$,

$$
\vDash \diamond \theta \rightarrow \theta^{w} \quad \text { and } \quad \vDash \theta^{s} \rightarrow \square \theta
$$

Indeed, note first that only the future operators $\mathbf{f}$ and $\mathbf{g}$ occur in $\theta^{w}$, i.e., every future operator is prefixed by a $\diamond$. Then, it suffices to use the validities $\vDash \diamond \alpha \vee \diamond \beta \leftrightarrow \diamond(\alpha \vee \beta), \vDash \diamond(\alpha \wedge \beta) \rightarrow \diamond \alpha \wedge \diamond \beta, \vDash \diamond F \alpha \rightarrow \diamond F \diamond \alpha$, and $\vDash \diamond G \alpha \rightarrow \diamond G \diamond \alpha$ to pull all $\diamond^{\prime} s$ in front of the formula and eventually show that $\vDash \diamond \theta \rightarrow \theta^{w}$. Likewise, but dually, for $\theta^{s}$.

A first consequence of (5.11) is that, for any Priorean formula $\theta$, tree T, valuation $V$ on $\mathrm{T}$, and $t \in T$ :

$$
h, V_{h}, t \vDash_{\text {lin }} \theta \text { for some } h \in \mathrm{H}_{t}(\mathrm{~T}) \quad \Rightarrow \quad \mathrm{T}, V, t \vDash_{\text {Peirce }} \theta^{w}
$$

and, taking into account that $\neg\left(\theta^{w}\right) \equiv(\neg \theta)^{s}$,

$$
\mathrm{T}, V, t \vDash_{\text {Peirce }} \theta^{s} \Rightarrow h, V_{h}, t \vDash_{\text {lin }} \theta \text { for every } h \in \mathrm{H}_{t}(\mathrm{~T})
$$

If bundled trees $\langle\mathrm{T}, \mathrm{B}\rangle$ are considered, it is straightforward to verify that the implications (5.12) and (5.13) became

$$
\begin{gathered}
h, V_{h}, t \vDash_{\text {lin }} \theta \text { for some } h \in \mathrm{B}_{t}(\mathrm{~T}) \quad \Rightarrow \quad\langle\mathrm{T}, \mathrm{B}\rangle, V, t \vDash_{\text {Peirce }} \theta^{w} \\
\langle\mathrm{~T}, \mathrm{~B}\rangle, V, t \vDash_{\text {Peirce }} \theta^{s} \Rightarrow h, V_{h}, t \vDash_{\text {lin }} \theta \text { for every } h \in \mathrm{B}_{t}(\mathrm{~T})
\end{gathered}
$$

Proposition 5.6 For any Priorean formula $\theta$ and any class of linear orders $\mathcal{C}$

$$
\mathcal{C} \vDash_{\text {lin }} \theta \quad \text { iff } \quad \mathcal{T}(\mathcal{C}) \vDash_{\text {Peirce }} \theta^{w}
$$

and

$$
\mathcal{C} \vDash_{\operatorname{lin}} \theta \quad \text { iff } \quad B^{+}(\mathcal{C}) \vDash_{\text {Peirce }} \theta^{w}
$$

Proof. In the first equivalence, the implication from right to left is immediate, because $\mathcal{C} \subseteq \mathcal{T}(\mathcal{C})$ and both semantics coincide on linear orders. 
Now, suppose $\mathcal{T}(\mathcal{C}) \not F_{\text {Peirce }} \theta^{w}$, i.e., $\mathrm{T}, V, t \vDash_{\text {Peirce }} \neg \theta^{w}$, and hence T, $V, t \vDash_{\text {Peirce }}$ $(\neg \theta)^{s}$, for some $\mathrm{T} \in \mathcal{T}(\mathcal{C}), V$, and $t$. Then (5.13) implies $h, V_{h}, t \vDash_{\text {lin }} \neg \theta$ for every $h \in \mathrm{H}_{t}(\mathrm{~T})$, but $\mathrm{H}_{t}(\mathrm{~T}) \subseteq \mathcal{C}$.

The second equivalence can be proved in the very same way, using $\left(5.13^{\prime}\right)$ instead of (5.13).

Let us consider again the density axiom $F p \rightarrow F F p$. The modal disjunctive normal form of it is $G \neg p \vee F F p$ and hence the weakest translation of this formula is $\mathbf{g} \neg p \vee \mathrm{ff} p$, equivalently $\mathbf{F} p \rightarrow \mathbf{f f} p$, while the strongest translation is $\mathbf{G} \neg p \vee \mathbf{F F} p$, equivalently $\mathbf{f} p \rightarrow \mathbf{F F} p$. We have already observed that none of these formulae defines density on trees, while each of the translations $\mathbf{f} p \rightarrow \mathbf{f f} p$ and $\mathbf{F} p \rightarrow \mathbf{F f} p$ does. The formula $\mathbf{f} p \rightarrow \mathbf{F F} p$ is actually not valid in the class of dense trees.

In general, the set of translations of a given Priorean formula $\theta$ can be endowed with a lattice structure by letting $\tau \leq \tau^{\prime}$ whenever $\tau(\theta)$ can be obtained from $\tau^{\prime}(\theta)$ by replacing some (possibly no) positive occurrence of $\mathbf{f}$ by $\mathbf{F}$ and some (possibly no) negative occurrence of $\mathbf{F}$ by $\mathbf{f}$. In this way $\tau^{w}$ and $\tau^{s}$ turn out to be the top and the bottom or the lattice, respectively. On the basis of the Peirce validity $\mathbf{F} \alpha \rightarrow \mathbf{f} \alpha$ it can be proved that

$$
\tau \leq \tau^{\prime} \Rightarrow \vDash_{\text {Peirce }} \tau(\theta) \rightarrow \tau^{\prime}(\theta) .
$$

The problem of characterizing the faithful syntactic translations is still partly open. In particular, a question arises if there is always a syntactic translation $\tau$ such that whenever $\theta$ defines the class $\mathcal{C}$ in the Priorean semantics, $\tau(\theta)$ defines $\mathcal{T}(\mathcal{C})$ in the Peircean semantics. The answer is 'no':

Example 5.7 Consider the classes $\mathcal{C}_{1}$ of dense linear orders and $\mathcal{C}_{2}$ of forwarddiscrete linear orders (where every point has an immediate successor, if any.) Let $\mathcal{C}=\mathcal{C}_{1} \cup \mathcal{C}_{2}$. Then $\alpha_{1}=F p \rightarrow F F p$ defines $\mathcal{C}_{1}$ and $\alpha_{2}=f \top \wedge q \wedge H q \rightarrow$ $\mathrm{FHq}$ defines $\mathcal{C}_{2}$. Moreover, it is easy to see that $\alpha_{1} \vee \alpha_{2}$ defines $\mathcal{C}$. On the other hand, it can be checked that none of the syntactic translations of $\alpha_{1} \vee \alpha_{2}$, nor even their conjunction, defines $\mathcal{C}$. Still, $\mathcal{C}$ is definable by the formula:

$$
\mathbf{G}\left(\left(\mathbf{H} \alpha_{1} \wedge \alpha_{1} \wedge \mathbf{G} \alpha_{1}\right) \vee\left((\mathbf{H H} \perp \vee \mathbf{P H} \mathbf{H} \perp) \wedge \mathbf{H}\left(\mathbf{P} \top \rightarrow \alpha_{2}\right) \wedge \alpha_{2} \wedge \mathbf{G} \alpha_{2}\right)\right) .
$$

The intuition is that in every tree from $\mathcal{T}(\mathcal{C})$, the dense and forward discrete branches must split already at the root into two disjoint subtrees. 


\section{Transfer of definability from linear orders to trees}

Note first that first-order definability is not preserved from a class of linear orders $\mathcal{C}$ to the class of trees $\mathcal{T}(\mathcal{C})$. For example, the property 'Every path has a last element' is not FO definable on trees: consider the complete infinite binary tree extended with last points for every path. It is uncountable, and must have a countable elementary subtree.

In this section we investigate the transfer of definability of a class of linear order $\mathcal{C}$ by means of a Priorean formula to definability of the associated classes of trees from $\mathbb{T}(\mathcal{C})$ in each of the Priorean, Peircean, and Ockhamist languages.

Given a Priorean formula $\psi$, we denote by $\operatorname{LIN}(\psi)$ the class of linear orders defined (within the class of all linear orders $\mathcal{C}_{\text {lin }}$ ) by $\psi$. Likewise, for any Ockhamist formula $\psi$ we denote by $\operatorname{TR}(\psi)$ and $\operatorname{BTR}(\psi)$ respectively the classes of trees and bundled trees defined by $\psi$.

\subsection{Transfer of definability to closures of classes of lin- ear orders}

The following (still partly open) question is of importance for what follows: if $\mathcal{C}=\operatorname{LIN}(\psi)$, can we define (uniformly) the class $\mathcal{C}^{*}$ in a linear time temporal language (possibly with Since and Until)? The answer is 'yes' provided the class $\operatorname{IS}(\mathcal{C})$ of proper initial segments of orders from $\mathcal{C}$, or even the class $\operatorname{IES}(\mathcal{C})$ of proper initial segments with endpoints from $\operatorname{IS}(\mathcal{C})$, is definable.

Observe first, indeed, that the bundle condition that every point in a tree $\mathrm{T}$ belongs to some path in any bundle on $\mathrm{T}$ implies that, for every class $\mathcal{C}$ of linear orders, $\operatorname{IS}(\mathcal{C})=\operatorname{IS}\left(\mathcal{C}^{*}\right)$ and $\operatorname{IES}(\mathcal{C})=\operatorname{IES}\left(\mathcal{C}^{*}\right)$. This means that the class $\mathcal{C}^{*}$ can also be defined as the class of all linear orders whose initial segments belong to $\operatorname{IS}(\mathcal{C})$ (or $\operatorname{IES}(\mathcal{C})$ ). Therefore, if the formula $\varphi$ defines, e.g., $\operatorname{IS}(\mathcal{C})$, then $\mathcal{C}^{*}$ is the class of linear orders $\mathrm{L}$ such that $\varphi$ is true in every proper initial segment of $\mathrm{L}$. The further step of the proof is to observe that, if $\mathrm{L}, V \models P F \pi \wedge P F \neg \pi \wedge H G(\pi \rightarrow H \pi)$ for a given propositional variable $\pi$, then $V(\pi)$ is a proper, non-empty, initial segment of L. Thus, finally, the 
elements of $\mathcal{C}^{*}$ are the linear orders $\mathrm{L}$ such that

$$
\mathrm{L} \models(P F \pi \wedge P F \neg \pi \wedge H G(\pi \rightarrow H \pi)) \rightarrow \varphi_{\pi}
$$

where the variable $\pi$ does not occur in $\varphi$ and $\varphi_{\pi}$ is the Priorean relativization of $\varphi$ to $\pi$, that is the formula inductively defined by means of the following clauses:

- $p_{i \pi}=\pi \wedge p_{i}$

- $(\neg \psi)_{\pi}=\neg \psi_{\pi} ; \quad\left(\psi \wedge \psi^{\prime}\right)_{\pi}=\psi_{\pi} \wedge \psi_{\pi}^{\prime} ;$

- $(P \psi)_{\pi}=P\left(\pi \wedge \psi_{\pi}\right) ; \quad(F \psi)_{\pi}=F\left(\pi \wedge \psi_{\pi}\right)$.

A more detailed proof of this kind will be given below, in Section 6.3, where definability in Peircean language will be considered.

\subsection{Transfer of definability to the Priorean language}

Let $\mathcal{C}=\operatorname{LIN}(\psi)$ for some linear time temporal formula $\psi$. Is the class $\mathcal{T}(\mathcal{C})$ always definable by a Priorean temporal formula? The answer is 'no'. For instance, $\mathcal{C}_{\text {last }}=\operatorname{LIN}(G \perp \vee F G \perp)$, while the class $\mathcal{T}\left(\mathcal{C}_{\text {last }}\right)$ is not definable by a Priorean formula, as a consequence from the following result.

Proposition 6.1 Let $\mathrm{T}_{1}$ be the tree obtained from the tree $\mathrm{T}_{0}$ of Figure 1 by adding a terminal point after the unique $\omega$-path in it. Then every Priorean formula $\theta$ is satisfiable in $T_{1}$ iff it is satisfiable in $T_{0}$.

Proof. For convenience, we assume that $T_{0} \subset T_{1}$. Let us fix a Priorean formula $\theta$, of modal depth $k$. Hereafter we restrict the language to only those variables that occur in $\theta$.

We will prove both directions by building for every pointed Kripke model on the one tree a suitable pointed Kripke model on the other, bisimilar to the former up to the modal depth $k$ of the formula $\theta$. For that purpose it suffices to ensure that the same types of depths up to $k$ are realized in both models.

1. Suppose first, that $\left(T_{0}, V_{0}\right), w \vDash \theta$ for some valuation $V_{0}$ and point $w$. Let $\chi_{1}^{k}, \ldots, \chi_{s}^{k}$ be all characteristic formulae of depth $k$ (see e.g., [10]) in 
the Priorean temporal language over the variables in $\theta$, realized at terminal points of $\left(T_{0}, V_{0}\right)$. At least one of these formulae, say $\chi_{1}^{k}$, will be realized in a cofinal set $E_{0}$ of terminal points of $T_{0}$. We define a valuation $V_{1}$ in $T_{1}$ as follows: $V_{1}$ coincides with $V_{0}$ on $T_{0}$, and is defined at the new point $\infty$ in accordance with $\chi_{1}^{k}$. We now claim that the relation between $\left(T_{1}, V_{1}\right)$ and $\left(T_{0}, V_{0}\right)$, which associates every point of $\left(T_{0}, V_{0}\right)$ to itself, and the point $\infty$ to all points in $\left(T_{0}, V_{0}\right)$ realizing $\chi_{1}^{k}$, is a $k$-bisimulation (see [10]), and hence $w$ satisfies the same formulae of depth at most $k$ in $\left(T_{1}, V_{1}\right)$ and $\left(T_{0}, V_{0}\right)$; in particular, $\left(T_{1}, V_{1}\right), w \vDash \theta$.

To prove that claim, it suffices to show, by induction on $m$, that for every $m \leq k$, every point from $T_{0}$ has the same type of depth $m$ in $T_{0}$ and in $T_{1}$, while the type of depth $m$ of $\infty$ is the same as the type of depth $m$ of any point from a cofinal subset $E_{m}$ of $E$. When $m=0$ these holds by definition. Suppose $m<k$ and the claim above holds for $m$. To prove it for $m+1$ we consider first $\infty$. Its type of depth $m+1$ looks like this:

$$
\chi_{1}^{0} \wedge \mathbf{G} \perp \wedge \mathbf{P} \chi_{1}^{m} \wedge \cdots \wedge \mathbf{P} \chi_{r}^{m} \wedge \mathbf{H}\left(\chi_{1}^{m} \vee \cdots \vee \chi_{r}^{m}\right)
$$

where $\chi_{1}^{m}, \cdots, \chi_{r}^{m}$ are all types of depth $m$ realized at predecessors of $\infty$. This case follows from the inductive hypothesis, and the fact that cofinally many points in $E_{m}$ have the same types of depth $m$ of predecessors as $\infty$; let that subset be $E_{m+1}$. The argument for every terminal point in $T_{0}$ is immediate from the inductive hypothesis; for every other point $u$ in $T_{0}$, the inductive claim follows from the inductive hypothesis, and the fact $u$ has (cofinally many) successors in $E_{m}$, so the type of depth $m$ of the successor $\infty$ of $u$ makes no difference to the type of depth $m+1$ of $u$ in $T_{1}$.

2. Now, suppose that $\left(T_{1}, V_{1}\right), w \vDash \theta$ for some valuation $V_{1}$ and point $w$. Let the non-terminal points in $T_{1}$ be $\left\{u_{1}, u_{2}, \ldots\right\}$, and the terminal points be respectively $\left\{v_{1}, v_{2}, \ldots\right\}$ and $\infty$.

First, we identify an index $n$ such that all types of depth $k$ of $u$-points in $T_{1}$ occur amongst $\left\{u_{1}, u_{2}, \ldots u_{n}\right\}$. Then, we select an infinite sequence of indices $\left\{n_{1}, n_{2}, \ldots\right\}$ such that:

C1) $n \leq n_{1}$.

C2) All $u_{n_{i}}$ 's have the same type of depth $k$ in $T_{1}$.

C3) All $v_{n_{i}}$ 's have the same type of depth $k$ in $T_{1}$. 
Such an infinite sequence exists, because there are only finitely many types of depth $k$ in $T_{1}$.

Now, we define a valuation $V_{0}$ in $T_{0}$ as follows: for every even $i$, we define $V_{0}$ at $v_{n_{i}}$ to be the same as $V_{1}$ at $\infty$; on all other points in $T_{0}$ we define $V_{0}$ to coincide with $V_{1}$.

We will prove the following by simultaneous induction on $m \leq k$ :

1) All $v_{n_{i}}$ 's, where $i$ is even, have the same type of depth $m$ in $T_{0}$ as $\infty$ in $T_{1}$.

2) All other points in $T_{0}$ have the same type of depth $m$ in $T_{0}$ as in $T_{1}$.

When $m=0$ the claims follow by definition. Suppose the claims hold for some $m<k$. We will show that they hold for $m+1$. First, the claim holds for the types of depth $m+1$ of all $v_{n_{i}}$ for even $i$ 's because, by condition C1 above and the inductive hypothesis, every such point has the same types of depth $m$ of predecessors in $T_{0}$ as $\infty$ in $T_{1}$. Likewise, the claim holds for all points in $T_{0}$ by condition $\mathrm{C} 2$ and $\mathrm{C} 3$ above, and the inductive hypothesis, as they will have respectively the same types of depth $m$ of predecessors and successors in $T_{0}$ as $\infty$ in $T_{1}$.

In particular, $\theta$ is satisfied in $T_{0}$ at any point with the same type of depth $k$ as the type of $w$ in $T_{1}$.

Consequently, we can show that definability does not transfer to any of the seven classes of trees and bundled trees associated with a class of linear orders $\mathcal{C}$.

\subsection{Transfer of definability to the Peircean language}

First, let us note that, as a consequence of Lemma 5.11, if $\theta$ defines the property $\mathcal{P}$ in the class of linear orders, then, for every tree $\mathrm{T}$ :

(1) $\mathrm{T}, t \vDash_{\text {Peirce }} \theta^{w}$ if a path passing through $t$ has the property $\mathcal{P}$, and

(2) T, $t \vDash_{\text {Peirce }} \theta^{s}$ only if every path passing through $t$ has $\mathcal{P}$.

As seen earlier, however, neither the weakest, nor the strongest Peircean translation is always suitable to define the class of trees $\mathcal{T}(\mathcal{C})$ for any class of linear orders $\mathcal{C}$ definable in the linear time temporal language; the former is generally too weak, while the latter is too strong. Still, it turns out that 
for all classes of linear orders $\mathcal{C}$ listed in Section 2, the respective classes of trees $\mathcal{T}(\mathcal{C})$ are definable in the Peircean language.

\subsubsection{Some examples}

Here we give defining formulae for some of the more interesting cases. We provide a detailed proof for two of them and leave the verification of the others to the reader.

Density: Either of $\mathbf{f} p \rightarrow \mathbf{f f} p$ and $\mathbf{F} p \rightarrow \mathbf{F f} p$.

Discreteness: $(\mathbf{f} \top \rightarrow((p \wedge \mathbf{H} p) \rightarrow \mathbf{F H} p)) \wedge(\mathbf{P} \top \rightarrow((p \wedge \mathbf{g} p)) \rightarrow \mathbf{P g} p)$.

Dedekind Continuity: $(\mathbf{F G} \neg p \wedge \mathbf{F} p) \rightarrow \mathbf{F}(\mathbf{g} \neg p \wedge \mathbf{H f} p)$.

Proof. Consider a tree $\mathrm{T}$ in which every path is Dedekind continuous and assume T, $V, x \models \mathbf{F G} \neg p \wedge \mathbf{F} p$. We have to show that any path $h$ passing through $x$ contains a point $x_{0}$ such that $x<x_{0}$ and $\mathrm{T}, V, x_{0}=$ $\mathbf{g} \neg p \wedge \mathbf{H f} p$. It can be easily verified that $x_{0}$ is the greatest lower bound of the set of all $y$ in $h$ such that $\mathrm{T}, V, y \models \mathbf{G} \neg p$.

Conversely assume that the tree $\mathrm{T}$ contains a path $h_{0}$ on which Dedekind continuity fails, that is: $h_{0}=X_{1} \cup X_{2}$, for all $x_{1} \in X_{1}$ and $x_{2} \in X_{2}$, $x_{1}<x_{2}$, but $X_{1}$ has no last element and $X_{2}$ has no first element. We can consider two different cases; in them, $X_{1}^{<}$denotes the set $\left\{x: x>x_{1}\right.$ for all $\left.x_{1} \in X_{1}\right\}$ (so that $X_{2} \subseteq X_{1}^{<}$).

Case 1: there exists an element $x_{0}$ of $X_{1}$ such that every path passing through $x_{0}$ has non-empty intersection with $X_{1}^{<}$(which means that there is no branching of paths in the future of $x_{0}$ on $\left.X_{1}\right)$. In this case, we let $V$ be any evaluation such that $X_{1} \subseteq V(p)$ and $V(p) \cap X_{1}^{<}=\emptyset$. Every path through $x_{0}$ meets a point in $X_{1}$ as well as point in $X_{1}^{<}$, and hence $\mathrm{T}, V, x_{0}=\mathbf{F G} \neg p \wedge \mathbf{F} p$. At any point $x$ of $h_{0} \cap\left\{y: y \geq x_{0}\right\}$, though, we have either T, $V, x \models \neg \mathbf{g} \neg p$ (if $x \in X_{1}$ ), or T, $V, x \models \neg \mathbf{H f} p$ (if $\left.x \in X_{2}\right)$. Thus, T, $V, x_{0} \not \models \mathbf{F}(\mathbf{g} \neg p \wedge \mathbf{H f} p)$.

Case 2: for every $x \in X_{1}$ there exists a path $h$ through $x$ such that $h \cap X_{1}^{<}=\emptyset$ (which implies that $h \backslash X_{1}$ has at least one element). Let 
$x_{0}$ be any fixed element of $X_{1}$ and let $V$ be any valuation on $\mathrm{T}$ with the following properties: 1) $\left.X_{1} \subseteq V(p), 2\right) V(p) \cap X_{1}^{<}=\emptyset$, and 3) for every path $h$ passing through $x_{0}$ and such that $h \cap X_{1}^{<}=\emptyset$, there are points $x_{h} \leq x_{h}^{\prime}$ in $h \backslash X_{1}$ such that $x_{h} \in V(p)$ and T, $V, x_{h}^{\prime} \models \mathbf{G} \neg p$. With this valuation, we have $\mathrm{T}, V, x_{0} \models \mathbf{F G} \neg p \wedge \mathbf{F} p$. However, for any $x>x_{0}$ in $h_{0}$, we have $\mathrm{T}, V, x \forall \mathbf{g} \neg p \wedge \mathbf{H f} p$.

$\omega$-trees: The conjunction of $\psi_{1}=\mathbf{H} \perp \vee \mathbf{P H} \perp, \psi_{2}=\mathbf{G} p \rightarrow \mathbf{F} p, \psi_{3}=$ $\mathbf{H}(\mathbf{H} p \rightarrow p) \rightarrow(\mathbf{P H} p \rightarrow \mathbf{H} p)$, and either of $\psi_{4}=\mathbf{G}(\mathbf{g} p \rightarrow p) \rightarrow$ $(\mathbf{f g} p \rightarrow \mathbf{g} p)$ and $\psi_{4}^{\prime}=\mathbf{g}(\mathbf{g} p \rightarrow p) \rightarrow(\mathbf{F G} p \rightarrow \mathbf{g} p)$.

Remark: the formula $\mathbf{G}(\mathbf{G} p \rightarrow p) \rightarrow(\mathbf{F G} p \rightarrow \mathbf{G} p)$ is valid in all $\omega$ trees, but also in a tree with a branch $\omega+\omega$ and $\omega$-branches stemming from every node of the $\omega$-initial segment of that branch.

Proof. It is readly verified that $\psi_{1}$ to $\psi_{4}$ (or $\psi_{4}^{\prime}$ ) are valid in every $\omega$-tree.

Consider now any tree $\mathrm{T}$ and assume that these formulae are valid in it. Formula $\psi_{1}$ guarantees that $\mathrm{T}$ has a first point, and by $\psi_{2}$ no path in $\mathrm{T}$ has a last point. Moreover, if $x_{0}>x_{1}>\ldots$ is an infinite descending sequence in $\mathrm{T}$, then we have $\mathrm{T}, V, x_{0} \models \neg \psi_{3}$ for any valuation $V$ such that $V(p)=\left\{x \in \mathrm{T}: \forall i\left(x_{i}>x\right)\right\}$. Thus, $\mathrm{T}$ has no infinite descending chain.

Finally, consider the formula $\psi_{4}$ and suppose that some infinite ascending chain $x_{0}<x_{1}<\ldots$ in $\mathrm{T}$ has an upper bound, say $x^{*}$. Consider a path $h^{*}$ passing through $x^{*}$ (and hence containing each $x_{i}$ ) and let $V$ be any valuation such that $V(p)=\left\{x \in h^{*}: \forall i\left(x_{i}<x\right)\right\}$. With this valuation we have T, $V, x_{0} \models \neg \psi_{4}$ and hence we can conclude that every infinite ascending chain in $\mathrm{T}$ is cofinal in some path. Therefore, every path in $\mathrm{T}$ has the order type of $\omega$.

$(\omega+1)$-trees: The conjunction of $\mathbf{H} \perp \vee \mathbf{P H} \perp, \mathbf{G} p \rightarrow \mathbf{F} p, \mathbf{H}(\mathbf{H} p \rightarrow p) \rightarrow$ $(\mathbf{P H} p \rightarrow \mathbf{H} p)$, and either of $\mathbf{G}(\mathbf{g} p \rightarrow p) \rightarrow(\mathbf{f}(\mathbf{f} \top \wedge \mathbf{g} p) \rightarrow \mathbf{g} p)$ and $\mathbf{g}(\mathbf{g} p \rightarrow p) \rightarrow(\mathbf{F}(\mathbf{f} \top \wedge \mathbf{G} p) \rightarrow \mathbf{g} p)$.

Z-trees: The conjunction of $\mathbf{H} p \rightarrow \mathbf{P} p, \mathbf{G} p \rightarrow \mathbf{F} p, \mathbf{H}(\mathbf{H} p \rightarrow p) \rightarrow(\mathbf{P H} p \rightarrow$ 
$\mathbf{H} p)$, and either of $\mathbf{G}(\mathbf{g} p \rightarrow p) \rightarrow(\mathbf{f g} p \rightarrow \mathbf{g} p)$ and $\mathbf{g}(\mathbf{g} p \rightarrow p) \rightarrow$ $(\mathbf{F G} p \rightarrow \mathbf{g} p)$.

Well-founded trees: $\mathbf{H}(\mathbf{H} p \rightarrow p) \rightarrow \mathbf{H} p$.

Conversely well-founded trees: $\mathbf{G}(\mathrm{g} p \rightarrow p) \rightarrow \mathbf{g} p$.

\subsubsection{Uniform translation}

A natural question arises now: is there a uniform translation into the Peircean language that can be used to define the class $\mathcal{T}(\mathcal{C})$ for any definable class of linear orders $\mathcal{C}$ ? Here we give an affirmative answer by providing such translation.

Let $\pi$ be a fixed, reserved propositional variable which does not belong to the Priorean temporal language on linear orders. For any Peircean formula $\varphi$, we define the $\pi$-relativization $\varphi_{\pi}$ of $\varphi$ by induction on the complexity of $\varphi$ as follows.

- $p_{i \pi}=\pi \wedge p_{i}$

- $(\neg \varphi)_{\pi}=\neg \varphi_{\pi} ; \quad\left(\varphi \wedge \varphi^{\prime}\right)_{\pi}=\varphi_{\pi} \wedge \varphi_{\pi}^{\prime} ;$

- $(\mathbf{P} \varphi)_{\pi}=\mathbf{P}\left(\pi \wedge \varphi_{\pi}\right) ; \quad(\mathbf{f} \varphi)_{\pi}=\mathbf{f}\left(\pi \wedge \varphi_{\pi}\right) ;$

- $(\mathbf{F} \varphi)_{\pi}=\mathbf{F}\left(\pi \wedge \mathbf{H} \pi \wedge \mathbf{g} \pi \rightarrow \varphi_{\pi}\right)$.

Lemma 6.2 Assume that $V$ is any evaluation on the tree $\mathrm{T}$ such that $\mathrm{T}, V, x \models$ $\operatorname{Pf} \pi \wedge \mathbf{H G}(\pi \rightarrow \mathbf{H} \pi \wedge \mathbf{g} \pi)$ for some $x$ in $\mathrm{T}$. Then:

1) $V(\pi)$ is a nonempty subtree $\mathrm{T}^{\prime}$ of $\mathrm{T}$ (hence, every path in $\mathrm{T}^{\prime}$ is a path in $\mathrm{T}$, too), and

2) for every Peircean formula $\varphi$ and every $y$ in $\mathrm{T}^{\prime}$ :

$$
\mathrm{T}, V, y=\varphi_{\pi} \quad \text { iff } \quad \mathrm{T}^{\prime}, V^{\prime}, y \models \varphi
$$

where $V^{\prime}$ is the restriction of $V$ to $\mathrm{T}^{\prime}$.

Proof. The first claim is obvious. The second one can easily be proved by induction on the complexity of $\varphi$. When $\varphi$ is $\mathbf{F} \psi$, we have to observe that 
the formula $\pi \wedge \mathbf{H} \pi \wedge \mathbf{g} \pi$ is true under $V$ at all points of a path $h$ in $\mathrm{T}$ if and only if $h$ is a path in $T^{\prime}$.

For any Priorean formula $\psi$, we define

$$
\psi^{T}:=(\mathbf{P f} \pi \wedge \mathbf{H G}(\pi \rightarrow \mathbf{H} \pi \wedge \mathbf{g} \pi)) \rightarrow \psi_{\pi}^{w},
$$

Theorem 6.3 For any class of linear orders $\mathcal{C}$ and any linear time formula $\psi$, the following are equivalent:

$$
\begin{aligned}
& (1) \quad \mathcal{C}=\operatorname{LIN}(\psi) \\
& (2) \mathcal{T}(\mathcal{C})=\operatorname{TR}\left(\psi^{T}\right) \\
& (3) \mathcal{B}^{+}(\mathcal{C})=\operatorname{BTR}\left(\psi^{T}\right)
\end{aligned}
$$

Moreover, if the class $\mathcal{C}$ is closed, then the following are equivalent:

$$
\begin{aligned}
& (1) \mathcal{C}=\operatorname{LIN}(\psi) \\
& (2) \mathcal{T}^{*}(\mathcal{C})=\mathcal{T}\left(\mathcal{C}^{*}\right)=\operatorname{TR}\left(\psi^{T}\right) \\
& (3) \\
& \mathcal{B}(\mathcal{C})=\mathcal{B}^{*}(\mathcal{C})=\mathcal{B}\left(\mathcal{C}^{*}\right)=\operatorname{BTR}\left(\psi^{T}\right)
\end{aligned}
$$

Proof. Consider first the equivalence $(1) \Leftrightarrow(2)$ in (6.16). The direction from right to left is rather easy, since $\mathcal{C} \subseteq \mathcal{T}(\mathcal{C})$ and $\mathrm{L} \vDash \psi$ iff $\mathrm{L} \vDash \psi^{T}$ for any linear order L considered as a tree.

For the other direction, assume $\mathcal{C}=\operatorname{LIN}(\psi)$ and first suppose $\mathrm{T} \vDash \psi^{T}$. If $\mathrm{T} \notin \mathcal{T}(\mathcal{C})$, then some path $h$ in $\mathrm{T}$ is not in $\mathcal{C}$ and hence $h$ falsifies $\psi$ for some valuation $V^{\prime}$ at some point $x$. Observe that, viewing $h$ as a tree, we have also $h, V^{\prime}, x \models \neg \psi^{w}$. Let $V$ be any extension of $V^{\prime}$ to $\mathrm{T}$ which makes $\pi$ true precisely on the points from $h$. Then, by Lemma 6.2 , T, $V, x \models \neg \psi_{\pi}^{w}$, which contradicts $\mathrm{T} \vDash \psi^{T}$ because $\mathrm{T}, V, x \models \mathbf{P f} \pi \wedge \mathbf{H G}(\pi \rightarrow \mathbf{H} \pi \wedge \mathbf{g} \pi)$.

Conversely, suppose $\mathrm{T} \not \models \psi^{T}$. Then T, $V, x \not \models \psi^{T}$, namely, T, $V, x \vDash \operatorname{Pf} \pi \wedge$ $\mathbf{H G}(\pi \rightarrow \mathbf{H} \pi \wedge \mathbf{g} \pi) \wedge \neg \psi_{\pi}^{w}$, for some valuation $V$ and point $x$. Thus $V(\pi)$ is the union of a non-empty set of paths in $\mathrm{T}$ and is a subtree $\mathrm{T}^{\prime}$ of $\mathrm{T}$. By Lemma 6.2, we have $\mathrm{T}^{\prime}, V^{\prime}, x \vDash \neg \psi^{w}$, where $V^{\prime}$ is the restriction of $V$ to $\mathrm{T}^{\prime}$, and hence, by (5.14) Proposition 5.6, $\mathrm{T}^{\prime} \notin \mathcal{T}(\mathcal{C})$. This implies that $\mathrm{T}$ does not belong to $\mathcal{T}(\mathcal{C})$ as well.

The proof of the equivalence $(1) \Leftrightarrow(3)$ in (6.16) is quite similar and uses (5.14') in Proposition 5.6.

The equivalence of (1), (2), and (3) in (6.17) is a consequence of the previous result and of the third claim in Theorem 3.10. 
Corollary 6.4 For any class of linear orders $\mathcal{C}$ and any Priorean formula $\psi$ :

$$
\mathcal{C} \vDash_{\text {lin }} \psi \quad \text { iff } \mathcal{T}(\mathcal{C}) \vDash_{\text {Peirce }} \psi^{T} \quad \text { iff } \quad \mathcal{B}^{+}(\mathcal{C}) \vDash_{\text {Peirce }} \psi^{T} \text {. }
$$

For the Peircean definability of $\mathcal{T}\left(\mathcal{C}^{*}\right)$ and $\mathcal{B}\left(\mathcal{C}^{*}\right)$ the following proposition slightly strengthen the second part of Theorem 6.3 .

Proposition 6.5 For any class $\mathcal{C}$ of linear orders, the classes $\mathcal{T}\left(\mathcal{C}^{*}\right)$ and $\mathcal{B}\left(\mathcal{C}^{*}\right)$ are Peirce definable if and only if the class $\mathcal{C}^{*}$ is Prior definable.

Proof. Assume that $\mathcal{C}^{*}$ is Prior definable. The Peircean definability of $\mathcal{T}\left(\mathcal{C}^{*}\right)$ and $\mathcal{B}\left(\mathcal{C}^{*}\right)$ is a consequence of Theorem 6.3 , taking into account that $\mathcal{C}^{*}$ is closed.

Assume now that $\mathcal{T}\left(\mathcal{C}^{*}\right)$ is Peirce definable by the formula $\varphi$. Then, for every linear order $\mathrm{L}$, viewed as a tree, we have $\mathrm{L}=\varphi$ iff $\mathrm{L} \in \mathrm{T}\left(\mathcal{C}^{*}\right)$ iff $\mathrm{L} \in \mathcal{C}^{*}$. Let $\varphi^{-}$be the formula obtained from $\varphi$ by replacing $\mathbf{f}$ and $\mathbf{F}$ by $F, \mathbf{g}$ and $\mathbf{G}$ by $G$, and $\mathbf{P}$ by $P$. Since Prior and Peircean semantics coincide on linear orders, by the equivalence above we have also $\mathrm{L}=\varphi^{-}$iff $\mathrm{L} \in \mathcal{C}^{*}$, and hence $\mathcal{C}^{*}$ is Prior definable.

The Prior definability of $\mathcal{C}^{*}$ under the hypothesis that $\mathcal{B}\left(\mathcal{C}^{*}\right)$ is Peirce definable can be proved in the same way, taking into account that linear orders, viewed as trees, have only the trivial bundle.

Thus, we see that the Peircean language suffices to match on trees the expressiveness of the Priorean language on linear orders.

Finally, we list a few facts regarding the classes of trees $\operatorname{Tr}\left({ }_{-}\right)$and bundled trees $B \operatorname{Tr}\left({ }_{-}\right)$definable by means of the weakest, strongest, and faithful translation of a given Priorean formula into the Peircean language. The routine proofs are left to the reader.

Proposition 6.6 For any Priorean formula $\psi$, if $\mathcal{C}=\operatorname{LIN}(\psi)$ then the following hold:

1. $\operatorname{Tr}\left(\tau^{s}(\psi)\right) \subseteq \mathcal{T}(\mathcal{C}) \subseteq \operatorname{Tr}\left(\tau^{w}(\psi)\right)$.

2. $\mathrm{B} \operatorname{Tr}\left(\tau^{s}(\psi)\right) \subseteq \mathcal{B}(\mathcal{C}) \subseteq \mathrm{B} \operatorname{Tr}\left(\tau^{w}(\psi)\right)$.

3. $B(\mathcal{C}) \subseteq B \operatorname{Tr}\left(\psi^{T}\right) \subseteq B^{+}(\mathcal{C})$. 


\subsection{On the transfer of definability to the Ockhamist language}

Peircean language is (properly) contained in Ockhamist language, hence Theorem 6.3 implies the respective Ockhamist definability results, too. In fact, these results have direct and simple proofs, too. For instance, if $\mathcal{C}=\operatorname{LIN}(\psi)$, then clearly the Ockhamist formula $H \square G \psi$ defines the class of trees $\mathcal{T}(\mathcal{C})$.

Moreover, Proposition 6.5 has an obvious analogue for the Ockhamist language and hence, we have that $\mathcal{T}\left(\mathcal{C}^{*}\right)$ and $\mathcal{B}\left(\mathcal{C}^{*}\right)$ are Ockhamist definable if and only if the class $\mathcal{C}^{*}$ is Prior definable.

\subsection{Transfer of definability: summary}

The results on transfer of definability obtained in this section are summarized as follows:

\begin{tabular}{||c|c|c|c||}
\hline \hline & Priorean & Peircean & Ockhamist \\
\hline $\mathcal{T}(\mathcal{C})$ & - & + & + \\
\hline $\mathcal{B}(\mathcal{C})$ & - & + if $\mathcal{C}$ is closed & + if $\mathcal{C}$ is closed \\
\hline $\mathcal{B}^{+}(\mathcal{C})$ & - & + & + \\
\hline $\mathcal{T}^{*}(\mathcal{C})$ & - & + if $\mathcal{C}$ is closed & + if $\mathcal{C}$ is closed \\
\hline $\mathcal{B}^{*}(\mathcal{C})$ & - & + if $\mathcal{C}$ is closed & + if $\mathcal{C}$ is closed \\
\hline $\mathcal{T}\left(\mathcal{C}^{*}\right)$ & - & + iff $\mathcal{C}^{*}$ is Prior definable & + iff $\mathcal{C}^{*}$ is Prior definable \\
\hline $\mathcal{B}\left(\mathcal{C}^{*}\right)$ & - & + iff $\mathcal{C}^{*}$ is Prior definable & + iff $\mathcal{C}^{*}$ is Prior definable \\
\hline \hline
\end{tabular}

\section{Concluding remarks}

In this paper we have studied the transfer of satisfiability, validity and definability from classes of linear orders to associated classes of trees and bundled trees in a hierarchy of modal and temporal languages. Most of these questions have apparently been little, or not at all, studied so far, and some of them have turned out to be more subtle than what one might expect. While we have obtained a fairly detailed picture, a number of questions and topics remain open for further investigation. Here is a sample list: 
1. Some questions on semantics:

(a) For which classes $\mathcal{C}$ of linear orders are the Peircean semantics over $\mathcal{T}(\mathcal{C})$ and over $\mathcal{B}(\mathcal{C})$ equivalent?

This was proved to be the case for the class $\mathcal{C}_{\text {lin }}$ in [5]. On the other hand, we have seen that it is not true for $\{\mathbf{N}\}$.

(b) For which classes $C$ of linear orders are the Ockhamist semantics over $\mathcal{T}(C)$ and over $\mathcal{B}(C)$ equivalent?

The example from [4], given in Section 4.3.3, shows that this is not the case for $\mathcal{C}_{\text {lin }}$. Some results from [17] show that the same holds for other classes of trees like, e.g., the class of trees in which every path has cofinality type $\omega$, or the class of trees called cofinally jointed in that paper. Apart from trivial cases like $\mathcal{C}_{\text {fin }}$, though, no cases of positive answers are known to us.

2. Some questions on (transfer of) definability:

(a) Perhaps the most interesting, in the context of the present study, still open question on definability of classes of linear orders is whether the closure of every Prior definable class of linear orders is Prior definable, too. We suspect that the answer is 'yes' but there does not seem to be a uniform argument. A closer look at, and a suitable classification of, such closures might offer an easier avenue to that answer.

(b) With respect to transfer of semantics and definability, the Peircean language emerges as the most interesting case. Some of the more interesting still open questions with respect to transfer of definability to the Peircean language are:

- Describe the classes of trees and bundled trees that are defined by the weakest and the strongest Peircean translations of a given Priorean formula $\psi$, if $\operatorname{LIN}(\psi)=\mathcal{C}$ ?

- Describe the class $\operatorname{BTr}\left(\psi^{T}\right)$ of bundled trees definable by the formula $\psi^{T}$ for a given Priorean formula $\psi$ in terms of the class of linear orders $\mathcal{C}=\operatorname{LIN}(\psi)$. 
- Precisely which classes of trees and bundled trees in $\mathbb{T}(\mathcal{C})$ are definable in the Peircean language whenever $\mathcal{C}=\operatorname{LIN}(\psi)$ ?

\section{Acknowledgements}

This research was done within the Italy - South Africa Scientific and Technological Cooperation Programme, funded by the National Research Foundation of South Africa through research grant GUN 2072004, and by the Ministry of Foreign Affairs of Italy. We gratefully acknowledge the financial support from these institutions. We also thank the referee for some useful remarks and corrections.

\section{References}

[1] J. van Benthem. The Logic of Time. Kluwer Academic Publishers, Dordrecht, second edition, 1993.

[2] K. Bowen and D. de Jongh. Some complete logics for branched time, Part 1: Well-founded time, forward looking operators. Technical report, ILLC, 1986.

[3] J. Burgess. The unreal future. Theoria, 44:157-179, 1978.

[4] J. Burgess. Logic and time. J. of Symbolic Logic, 44:556-582, 1979.

[5] J. Burgess. Decidability for branching time. Studia Logica, 39:203-218, 1980.

[6] M. C. Di Maio and A. Zanardo. Synchronized histories in PriorThomason representation of branching time. In D. Gabbay and H. Ohlbach, editors, Proceedings of the First International Conference on Temporal Logic, pages 265-282. Springer-Verlag, 1994.

[7] E.A. Emerson. Temporal and modal logics. In J. van Leeuwen, editor, Handbook of Theoretical Computer Science, volume B: Formal Models and Semantics, pages 995-1072. Elsevier, 1990. 
[8] L. Gillman and M. Jerison. Rings of continuous functions. The University series in higher mathematics. Van Nostrand, Princeton, 1960.

[9] R. Goldblatt. Logics of Time and Computation, volume 7 of CSLI Lecture Notes. Center for the Study of Language and Information, Stanford University, 2nd. ed. edition, 1992.

[10] V. Goranko and M. Otto. Model theory of modal logic. In J. van Benthem, P. Blackburn, and F. Wolter, editors, Handbook of Modal Logic. Elsevier, 2006. To appear.

[11] A. Prior. Past, Present and Future. Clarendon, Oxford, 1967.

[12] J.G. Rosenstein. Linear Orderings. Academic Press, 1982.

[13] M. Sabbadin and A. Zanardo. Topological aspects of branching-time semantics. Studia Logica, 75:271-286, 2003.

[14] K. Segerberg. Modal logics with linear alternative relations. Theoria, 36:301-322, 1970.

[15] C. Stirling. Modal and temporal logics. In Handbook of Logic in Computer Science, volume 2 (Background: Computational Structures), pages 477-563. Clarendon Press, Oxford, 1992.

[16] A. Zanardo. Branching-time logic with quantification over branches: the point of view of modal logic. J. of Symbolic Logic, 61(1):1-39, 1996.

[17] A. Zanardo, B. Barcellan, and M. Reynolds. Non-definability of the class of complete bundled trees. Logic J. of the IGPL, 7(1):125-136, 1999. Special issue on Temporal Logic. 